\title{
Eigenvalue Problems on Domains with Cracks I
}

\author{
Kazushi YOSHITOMI
}

Tokyo Metropolitan University

\begin{abstract}
We study an eigenvalue problem for the Laplace operator on a planar region with a growing crack. We impose the Neumann boundary condition on the crack and the Dirichlet boundary condition elsewhere. One tip of the crack is fixed at the boundary. We obtain the full asymptotic expansions of the first two eigenvalues of that operator as the other tip of the crack reaches the boundary.
\end{abstract}

\section{Introduction}

In this paper we investigate the asymptotic expansions of the eigenvalues of the Laplace operator on a region with a growing crack.

Let $\Omega$ be a bounded, simply connected region in $\mathbf{R}^{2}$ with a smooth boundary and let $\gamma:\left[0, t_{0}\right] \rightarrow \mathbf{R}^{2}$ be a smooth curve without self-intersection. We assume that

$$
\gamma\left(\left(0, t_{0}\right)\right) \subset \Omega, \quad \gamma(0)=0 \in \partial \Omega, \quad \text { and } \quad \gamma\left(t_{0}\right) \in \partial \Omega
$$

For $\varepsilon \in\left[0, t_{0}\right)$, we put

$$
\Omega_{\varepsilon}=\Omega \backslash \gamma\left(\left[\varepsilon, t_{0}\right)\right) .
$$

For $b>0$, we define

$$
\Pi_{b}=((0, \infty) \times \mathbf{R}) \backslash([b, \infty) \times\{0\}) .
$$

For $a \in \mathbf{R}^{2}$ and $r>0$, we denote by $D(a, r)$ the open planar disk of radius $r$ centered at $\{a\}$. We impose the following assumption on $\Omega$ and $\gamma$.

(A.2) There exists $r_{0} \in\left(0, t_{0}\right)$ such that

$$
\Omega_{\varepsilon} \cap D\left(0, r_{0}\right)=\Pi_{\varepsilon} \cap D\left(0, r_{0}\right) \text { for all } \varepsilon \in\left[0, r_{0}\right] .
$$

The set $\Omega_{0}$ consists of two connected components. Let $\Omega_{+}$and $\Omega_{-}$be the connected components of $\Omega_{0}$ which satisfy $\left(0, r_{0} / 2\right) \in \partial \Omega_{+}$and $\left(0,-r_{0} / 2\right) \in \partial \Omega_{-}$, respectively. We define

$$
Q_{\varepsilon}=\text { the closure of }\left\{u \in C^{\infty}\left(\Omega_{\varepsilon}\right) \cap H^{1}\left(\Omega_{\varepsilon}\right) \mid \operatorname{supp} u \cap \partial \Omega=\emptyset\right\} \text { in } H^{1}\left(\Omega_{\varepsilon}\right)
$$

$$
Q^{ \pm}=\text {the closure of }\left\{u \in C^{\infty}\left(\Omega_{ \pm}\right) \cap H^{1}\left(\Omega_{ \pm}\right) \mid \operatorname{supp} u \cap \partial \Omega_{ \pm} \cap \partial \Omega=\emptyset\right\} \text { in } H^{1}\left(\Omega_{ \pm}\right),
$$




$$
\begin{gathered}
q_{\varepsilon}(u, v)=(\nabla u, \nabla v)_{L^{2}(\Omega)} \quad \text { for } \quad u, v \in Q_{\varepsilon}, \\
q^{ \pm}(u, v)=(\nabla u, \nabla v)_{L^{2}\left(\Omega_{ \pm}\right)} \quad \text { for } \quad u, v \in Q^{ \pm} .
\end{gathered}
$$

Let $L_{\varepsilon}$ be the self-adjoint operator associated with the quadratic form $q_{\varepsilon}$. The operator $L_{\varepsilon}$ is the negative Laplacian in $\Omega_{\varepsilon}$ subject to the Dirichlet boundary condition on $\partial \Omega$ and the Neumann boundary condition on the crack $\gamma\left(\left(\varepsilon, t_{0}\right)\right)$. By $\lambda_{j}(\varepsilon)$ we denote the $j$ th eigenvalue of $L_{\varepsilon}$ counted with multiplicity. The aim of this paper is to find the full asymptotic expansions of the first two eigenvalues of $L_{\varepsilon}$ as $\varepsilon$ tends to zero. Let $L^{+}$and $L^{-}$be the self-adjoint operators associated with the quadratic forms $q^{+}$and $q^{-}$, respectively. The operator $L^{ \pm}$is the negative Laplacian in $\Omega_{ \pm}$with the Dirichlet boundary condition on $\partial \Omega_{ \pm} \cap \partial \Omega$ and the Neumann boundary condition on $\gamma\left(\left(0, t_{0}\right)\right)$. Let $\lambda_{1}^{ \pm}<\lambda_{2}^{ \pm} \leq \lambda_{3}^{ \pm} \leq \cdots$ be the eigenvalues of $L^{ \pm}$repeated according to multiplicity. We confine our attention to the case where $\lambda_{1}^{+}=\lambda_{1}^{-}$, because the analysis of $\lambda_{1}(\varepsilon)$ in this case is more difficult than that in the case where $\lambda_{1}^{+} \neq \lambda_{1}^{-}$. We suppose that

$$
\lambda_{1}^{+}=\lambda_{1}^{-} .
$$

Let $\Psi_{0}^{ \pm}(x)$ be the eigenfunction of $L^{ \pm}$associated with the first eigenvalue $\lambda_{1}^{ \pm}$which is normalized by the conditions

$$
\Psi_{0}^{ \pm}(x)>0 \quad \text { in } \quad \Omega_{ \pm}, \quad\left\|\Psi_{0}^{ \pm}\right\|_{L^{2}\left(\Omega_{ \pm}\right)}=1 .
$$

By a simple reflection argument we see that $\Psi_{0}^{ \pm}$extends to an analytic function in $D\left(0, r_{0} / 2\right)$. Moreover, the function $\Psi_{0}^{ \pm}$is given by a convergent power series expansion:

$$
\begin{gathered}
\Psi_{0}^{ \pm}(x)=\sum_{j=1}^{\infty} \sum_{k=1}^{j} C_{j, k}^{ \pm} r^{2 j-1} \cos (2 k-1) \theta \quad \text { in } D\left(0, r_{0} / 2\right), \\
C_{1,1}^{ \pm}>0
\end{gathered}
$$

where $(r, \theta)$ stand for the polar coordinates of $x$ centered at $\{0\}$. Let

$$
K=\left\{\begin{array}{l|l}
j \geq 2 & \frac{C_{j, j}^{+}}{C_{1,1}^{+}} \neq \frac{C_{j, j}^{-}}{C_{1,1}^{-}}
\end{array}\right\} .
$$

We define $v=\min K$ if $K \neq \emptyset$. We put $\lambda_{0}=\lambda_{1}^{+}\left(=\lambda_{1}^{-}\right)$. Our main result is the following claim.

THEOREM 0.1. We have the assertions (i), (ii), and (iii) below.

(i) The second eigenvalue $\lambda_{2}(\varepsilon)$ admits the asymptotic expansion of the form

$$
\lambda_{2}(\varepsilon) \sim \lambda_{0}+\sum_{m=1}^{\infty} \sum_{n=0}^{m-1} \lambda_{m, n} \varepsilon^{2 m}(\log \varepsilon)^{n} \quad \text { as } \quad \varepsilon \rightarrow 0,
$$


where

$$
\lambda_{1,0}=\frac{\pi}{8}\left(\left(C_{1,1}^{+}\right)^{2}+\left(C_{1,1}^{-}\right)^{2}\right) .
$$

(ii) If $K \neq \emptyset$, then the first eigenvalue $\lambda_{1}(\varepsilon)$ has the asymptotic expansion of the form

$$
\lambda_{1}(\varepsilon) \sim \lambda_{0}+\sum_{i=2 v-1}^{\infty} \sum_{j=0}^{[(i-2 v+1) / 3]} \mu_{i, j} \varepsilon^{2 i}(\log \varepsilon)^{j} \quad \text { as } \quad \varepsilon \rightarrow 0
$$

with

$$
\mu_{2 v-1,0}>0 .
$$

(iii) If $K=\emptyset$, then

$$
\lambda_{1}(\varepsilon)=\lambda_{0}
$$

for sufficiently small $\varepsilon>0$.

This theorem implies that if $K \neq \emptyset$, then $\lambda_{1}(\varepsilon)$ is increasing at $\varepsilon=0$; otherwise $\lambda_{1}(\varepsilon)=$ $\lambda_{0}$ for sufficiently small $\varepsilon>0$. Under some additional conditions on $\gamma$, we can determine whether $K \neq \emptyset$ or $K=\emptyset$ by the following theorem.

THEOREM 0.2. Suppose that there exists $\tau \in C^{\infty}\left(\left[0, t_{0}\right]\right)$ such that

$$
\gamma(t)=(t, \tau(t)) \quad \text { on }\left[0, t_{0}\right] \text { and } \tau\left(t_{0}\right)=0 .
$$

Then the statements (i) and (ii) below are equivalent.

(i) $K=\emptyset$.

(ii) The region $\Omega$ is symmetric with respect to the $x_{1}$-axis and $\tau(t)=0$ on $\left[0, t_{0}\right]$.

There are many works on the behavior of the eigenvalues of an elliptic boundary value problem on a region when a small deformation is turned on the region. In the case when the region is smoothly deformed, one can analyze the eigenvalues by using the Hadamard variation formula [6] or, more directly, Kato-Rellich's perturbation theory for abstract linear operators (see [9, Chapter VII, Section 6.5] and its references). The point of these arguments is to reduce the equation on the deformed region to an equation on the original region; the coefficients of the latter equation then depend smoothly on a deformation parameter. However, these arguments do not work in the case when the region is singularly deformed. To the best of the knowledge of the author, such a singular deformation was studied by A. M. Il'in [7] for the first time. He obtained the full asymptotic expansion of the harmonic function on a region with a narrow cavity by using the method of matched asymptotic expansion. The essence of this method is to decompose the region into overlapping subregions and to construct asymptotic solutions on the respective subregions so that the solutions asymptotically coincide on the intersection of the subregions. This method was fully developed by numerous authors; we refer to $[3,4,10]$ and $[8]$ for a thorough review. 
Our problem is also a singular deformation, because there is no diffeomorphism from $\Omega_{0}$ onto $\Omega_{\varepsilon}(\varepsilon>0)$. While we use the method of matched asymptotic expansions to prove the main theorem, our problem has the characteristic that the asymptotic form of the first eigenvalue heavily depends on the quantity $v=\min K$. This increases difficulties in seeking approximate solutions.

Our study is inspired and motivated by the work of M. Dauge and B. Helffer [1]. By using the method of variation, they proved (under more general assumptions than ours) that

$$
\lim _{\varepsilon \rightarrow 0} \lambda_{j}(\varepsilon)=v_{j} \text { for all } j \geq 1,
$$

where $v_{1} \leq v_{2} \leq \cdots$ are the rearrangement of $\left\{\lambda_{j}^{+}\right\}_{j=1}^{\infty} \cup\left\{\lambda_{j}^{-}\right\}_{j=1}^{\infty}$ counted with multiplicity. Our Theorems 0.1 and 0.2 fully extend their result for $j=1,2$.

We give the form of approximate solutions. We introduce the inner variable:

$$
\xi=\varepsilon^{-1} x
$$

We look for the approximate second eigenvalue of $L_{\varepsilon}$ and the associated approximate eigenfunctions in the following form.

$$
\begin{gathered}
\lambda(\varepsilon)=\lambda_{0}+\sum_{m=1}^{\infty} \sum_{n=0}^{m-1} \lambda_{m, n} \varepsilon^{2 m}(\log \varepsilon)^{n} . \\
\Psi_{\varepsilon}^{\text {out }, \pm}(x)=\varphi_{0,0}^{ \pm}(x)+\sum_{i=1}^{\infty} \sum_{j=0}^{i-1} \varepsilon^{2 i}(\log \varepsilon)^{j} \varphi_{i, j}^{ \pm}(x) \quad \text { in } \quad \Omega_{ \pm} \backslash D(0, \sqrt{\varepsilon}) . \\
\Psi_{\varepsilon}^{\text {in }}(x)=\sum_{k=1}^{\infty} \sum_{l=0}^{k-1} \varepsilon^{2 k-1}(\log \varepsilon)^{l} v_{k, l}(\xi) \quad \text { in } \quad \Omega_{\varepsilon} \cap D(0,2 \sqrt{\varepsilon}) .
\end{gathered}
$$

Here the leading term $\varphi_{0,0}^{ \pm}$on $(0.9)_{ \pm}$is a constant-multiple of $\Psi_{0}^{ \pm}$. We construct these approximate eigenfunctions in such a way that $\Psi_{\varepsilon}^{\text {in }}(x)$ asymptotically coincides with $\Psi_{\varepsilon}^{\text {out, }+}(x)$ and $\Psi_{\varepsilon}^{\text {out, }-}(x)$ on the intermediate regions $\Omega_{+} \cap(D(0,2 \sqrt{\varepsilon}) \backslash D(0, \sqrt{\varepsilon}))$ and $\Omega_{-} \cap(D(0,2 \sqrt{\varepsilon}) \backslash D(0, \sqrt{\varepsilon}))$, respectively. Inserting (0.8) and (0.9) \pm into the equation $\left(\Delta_{x}+\lambda(\varepsilon)\right) \Psi_{\varepsilon}^{\text {out, } \pm}(x)=0$ and identifying the powers of $\varepsilon$ and $\log \varepsilon$, we have the outer equations

$$
\left\{\begin{array}{l}
\left(\Delta_{x}+\lambda_{0}\right) \varphi_{p, q}^{ \pm}=-\lambda_{p, q} \varphi_{0,0}^{ \pm}-\sum_{m=1}^{p-1} \sum_{n=0}^{q} \lambda_{m, n} \varphi_{p-m, q-n}^{ \pm} \quad \text { in } \quad \Omega_{ \pm}, \\
\varphi_{p, q}^{ \pm}(x)=0 \quad \text { on } \quad \partial \Omega \cap \partial \Omega_{ \pm}, \quad \frac{\partial}{\partial n} \varphi_{p, q}^{ \pm}(x)=0 \quad \text { on } \quad \gamma\left(\left(0, t_{0}\right)\right) .
\end{array}\right.
$$


Plugging (0.8) and (0.10) into the equation $\left(\Delta_{x}+\lambda(\varepsilon)\right) \Psi_{\varepsilon}^{\text {in }}(x)=0$ and equating the powers of $\varepsilon$ and $\log \varepsilon$ on account of $\Delta_{x}=\varepsilon^{-2} \Delta_{\xi}$, we get the inner equations

$$
\left\{\begin{array}{l}
\Delta_{\xi} v_{p+1, q}=-\lambda_{0} v_{p, q}-\sum_{m=1}^{p-1} \sum_{n=0}^{q} \lambda_{m, n} v_{p-m, q-n} \text { in } \Pi_{1}, \\
v_{p+1, q}(\xi)=0 \quad \text { on }\{0\} \times \mathbf{R}, \quad \frac{\partial}{\partial \xi_{2}} v_{p+1, q}(\cdot, \pm 0)=0 \text { on }(1, \infty) .
\end{array}\right.
$$

We also seek the approximate first eigenvalue of $L_{\varepsilon}$ and the associated approximate eigenfunctions in the following form in the case when $K \neq \emptyset$.

$$
\begin{gathered}
\mu(\varepsilon)=\lambda_{0}+\sum_{i=2 v-1}^{\infty} \sum_{j=0}^{[(i-2 v+1) / 3]} \mu_{i, j} \varepsilon^{2 i}(\log \varepsilon)^{j}, \\
\psi_{\varepsilon}^{\mathrm{out}, \pm}(x)=\sum_{k=0}^{\infty} \sum_{l=0}^{[(k-v+2) / 3]} \varepsilon^{2 k}(\log \varepsilon)^{l} \psi_{k, l}^{ \pm}(x) \quad \text { in } \quad \Omega_{ \pm} \backslash D(0, \sqrt{\varepsilon}), \\
\psi_{\varepsilon}^{\text {in }}(x)=\sum_{i=1}^{\infty} \sum_{j=0}^{[(i-v+1) / 3]} \varepsilon^{2 i-1}(\log \varepsilon)^{j} w_{i, j}(\xi) \quad \text { in } \quad \Omega_{\varepsilon} \cap D(0,2 \sqrt{\varepsilon}) .
\end{gathered}
$$

Here the leading term $\psi_{0,0}^{ \pm}$of $(0.14)_{ \pm}$is also a constant-multiple of $\Psi_{0}^{ \pm}$. These approximate solutions are also constructed so that $\psi_{\varepsilon}^{\text {in }}(x)$ asymptotically coincides with $\psi_{\varepsilon}^{\text {out },+}(x)$ and $\psi_{\varepsilon}^{\text {out },-}(x)$ on the intermediate regions $\Omega_{+} \cap(D(0,2 \sqrt{\varepsilon}) \backslash D(0, \sqrt{\varepsilon}))$ and $\Omega_{-} \cap(D(0,2 \sqrt{\varepsilon}) \backslash D(0, \sqrt{\varepsilon}))$, respectively. As in the derivation of $(0.11)^{ \pm}$and $(0.12)$ we have

$$
\begin{aligned}
& \left\{\begin{aligned}
\left(\Delta_{x}+\lambda_{0}\right) \psi_{k, l}^{ \pm} & =-\sum_{i=3 l+2 v-1}^{k} \mu_{i, l} \psi_{k-i, 0}^{ \pm}-\sum_{j=0}^{l-1} \sum_{i=3 j+2 v-1}^{k-3 l+3 j-v+2} \mu_{i, j} \psi_{k-i, l-j}^{ \pm} \quad \text { in } \Omega_{ \pm}, \quad(0.16)_{ \pm} \\
\psi_{k, l}^{ \pm}=0 \quad \text { on } \quad \partial \Omega_{ \pm} \cap \partial \Omega, \quad \frac{\partial}{\partial n} \psi_{k, l}^{ \pm}=0 \quad \text { on } \quad \gamma\left(\left(0, t_{0}\right)\right), &
\end{aligned}\right. \\
& \left\{\begin{array}{l}
\Delta_{\xi} w_{s+1, q} \\
=-\lambda_{0} w_{s, q}-\sum_{i=3 q+2 v-1}^{s-1} \mu_{i, q} w_{s-i, 0}-\sum_{j=0}^{q-1} \sum_{i=3 j+2 v-1}^{s-3 q+3 j-v+1} \mu_{i, j} w_{s-i, q-j} \quad \text { in } \Pi_{1} \\
w_{s+1, q}=0 \quad \text { on } \quad\{0\} \times \mathbf{R}, \quad \frac{\partial}{\partial \xi_{2}} w_{s+1, q}(\cdot, \pm 0)=0 \quad \text { on } \quad(1, \infty) .
\end{array}\right.
\end{aligned}
$$


We organize this paper as follows. In section 1 we construct the solutions to the outer equations (0.11) and (0.16), and we analyze the asymptotic behavior of the solutions in a neighborhood of the origin. For the latter purpose we cannot directly utilize the general regularity theory of solutions to elliptic differential equations, because the region $\Omega_{ \pm}$has a conic singularity at the origin. We eliminate this difficulty by using a reflection argument, which enables us to apply the general regularity theory. In section 2 we construct the solutions to the inner equations (0.12) and (0.17), and we study the asymptotic behavior of the solutions in a neighborhood of infinity. To this end we reduce these equations to the Poisson equations in $\mathbf{R}^{2}$ by using a reflection argument and a conformal map. In section 3 we construct the coefficients of the approximate second eigenvalue (0.8) and those of the associated approximate eigenfunctions $(0.9)_{ \pm}$and $(0.10)$ which satisfy the mentioned coincidence on the intermediate regions by combining the results in sections 1 and 2 . In section 4 we construct an approximate eigenfunction in $\Omega_{\varepsilon}$ by joining the inner expansion $(0.10)$ to the outer ones $(0.9)_{ \pm}$, and we give an estimate on the error of the resulting appoximate eigenfunction. In section 5 we construct the approximate first eigenvalue $(0.13)$ and the associated approximate eigenfunctions $(0.14)_{ \pm}$and $(0.15)$, and we complete the proof of Theorem 0.1 . In section 6 we prove Theorem 0.2 by using analytic continuation. Section 7 is devoted to an appendix; we prove a density result in $H^{1}$-spaces which we need in sections 4 and 6 .

Throughout this paper we adopt the following conventions to reduce complicated classifications. We denote inessential constants by $C$ on estimations. For $k, l \in \mathbf{Z}$ with $k>l$, we define $\sum_{j=k}^{l} a_{j}=0$ and $\left\{b_{j}\right\}_{k \leq j \leq l}=\emptyset$. A formula which contains either \pm or $\mp$ means two formulae which correspond to the upper sign and the lower sign, respectively. For example, the formula $a^{ \pm}=b^{\mp}$ means that $a^{+}=b^{-}$and $a^{-}=b^{+}$. We also regard undefined terms as zero in formulae. For example, if $a$ and $c$ are defined and if $b$ is undefined, then the formula $a+b=c$ means $a=c$. We denote by $\mathbf{N}$ the set of all non-negative integers. We define $\mathbf{N}^{*}=\mathbf{N} \backslash\{0\}$.

\section{Outer equations}

In this section we solve the outer equations $(0.11)_{ \pm}$and $(0.16)_{ \pm}$by a reflection argument. As its preliminary we need the following claim.

Proposition 1.1. The eigenfunction $\Psi_{0}^{ \pm}$extends to a real analytic function in $D\left(0, r_{0} / 2\right)$ and admits convergent power-series expansion of the form (0.2) with (0.3).

Proof. We prove the assertion only for $\Psi_{0}^{+}$because that for $\Psi_{0}^{-}$is similar.

We proceed by the method of reflection. For $r>0$ we put

$$
\begin{aligned}
& D_{ \pm}(r)=\left\{\left(x_{1}, x_{2}\right) \in D(0, r) \mid \pm x_{1}>0\right\} \\
& D_{+ \pm}(r)=\left\{\left(x_{1}, x_{2}\right) \in D_{+}(r) \mid \pm x_{2}>0\right\} .
\end{aligned}
$$


We set

$$
\psi\left(x_{1}, x_{2}\right)=\left\{\begin{array}{lll}
\Psi_{0}^{+}\left(x_{1}, x_{2}\right) & \text { for } & x \in D_{++}\left(r_{0}\right) \\
\Psi_{0}^{+}\left(x_{1},-x_{2}\right) & \text { for } & x \in D_{+-}\left(r_{0}\right)
\end{array}\right.
$$

The function $\psi(x)$ is even in $x_{2}$. Because $\Psi_{0}^{+} \in Q^{+}$, we have

$$
\psi \in H^{1}\left(D_{+}\left(r_{0}\right)\right), \quad \psi=0 \quad \text { on } \quad\{0\} \times\left(-r_{0}, r_{0}\right) .
$$

Given $\phi \in C_{0}^{\infty}\left(D_{+}\left(r_{0}\right)\right)$, we set $\tilde{\phi}\left(x_{1}, x_{2}\right)=\phi\left(x_{1},-x_{2}\right)$ for $x \in D_{++}\left(r_{0}\right)$. Because $\Psi_{0}^{+}$is the eigenfunction of $L^{+}$associated with the eigenvalue $\lambda_{0}$, we have

$$
q_{0}^{+}\left(\Psi_{0}^{+}, v\right)=\lambda_{0}\left(\Psi_{0}^{+}, v\right)_{L^{2}\left(\Omega_{+}\right)} \quad \text { for any } \quad v \in Q^{+}
$$

Thus we obtain

$$
\begin{aligned}
\int_{D_{+}\left(r_{0}\right)} \psi(-\Delta \phi) d x & =\int_{D_{+}\left(r_{0}\right)} \nabla \psi \cdot \nabla \phi d x \\
& =\int_{D_{++}\left(r_{0}\right)} \nabla \psi \cdot \nabla \phi d x+\int_{D_{+-}\left(r_{0}\right)} \nabla \psi \cdot \nabla \phi d x
\end{aligned}
$$

by the coordinate change $\left(x_{1}, x_{2}\right) \mapsto\left(x_{1},-x_{2}\right)$ on the second term

$$
=\int_{D_{++}\left(r_{0}\right)} \nabla \Psi_{0}^{+} \cdot \nabla \phi d x+\int_{D_{++}\left(r_{0}\right)} \nabla \Psi_{0}^{+} \cdot \nabla \tilde{\phi} d x
$$

by using (1.2) and $\phi, \tilde{\phi} \in Q^{+}$

$$
=\lambda_{0} \int_{D_{++}\left(r_{0}\right)} \Psi_{0}^{+} \phi d x+\lambda_{0} \int_{D_{++}\left(r_{0}\right)} \Psi_{0}^{+} \tilde{\phi} d x
$$

by the coordinate change $\left(x_{1}, x_{2}\right) \mapsto\left(x_{1},-x_{2}\right)$ on the second term

$$
\begin{aligned}
& =\lambda_{0} \int_{D_{++}\left(r_{0}\right)} \psi \phi d x+\lambda_{0} \int_{D_{+-}\left(r_{0}\right)} \psi \phi d x \\
& =\lambda_{0} \int_{D_{+}\left(r_{0}\right)} \psi \phi d x .
\end{aligned}
$$

Thus we get

$$
-\Delta \psi=\lambda_{0} \psi \quad \text { in the sense of distribution in } D_{+}\left(r_{0}\right) .
$$

Using (1.1), (1.3), and the regularity estimate for the solutions of elliptic differential equations (see [2, Part 1, Section 17]), we obtain

$$
\psi \in H^{2}\left(D_{+}\left(r_{0} / 2\right)\right)
$$


Next we set

$$
\tilde{\psi}\left(x_{1}, x_{2}\right)= \begin{cases}\psi\left(x_{1}, x_{2}\right) & \text { for } \quad x \in D_{+}\left(r_{0} / 2\right) \\ -\psi\left(-x_{1}, x_{2}\right) & \text { for } \quad x \in D_{-}\left(r_{0} / 2\right)\end{cases}
$$

The function $\tilde{\psi}(x)$ is even in $x_{2}$ and is odd in $x_{1}$. By (1.1) we have $\tilde{\psi} \in H^{1}\left(D\left(0, r_{0} / 2\right)\right)$. As in the above observation, we deduce that

$$
-\Delta \tilde{\psi}=\lambda_{0} \tilde{\psi} \quad \text { in the sense of distribution in } D\left(0, r_{0} / 2\right) .
$$

Thus the regularity estimate for the solutions to elliptic differential equations (c.f. [2, Part 1, Section 15]) implies that

$$
\tilde{\psi} \in C^{\infty}\left(D\left(0, r_{0} / 2\right)\right) .
$$

Moreover, the analytic hypoellipticity for elliptic differential equations with analytic coefficients (see [2, Part3, Section1]) implies that $\tilde{\psi}$ is real analytic in $D\left(0, r_{0} / 2\right)$. Combining this with the fact that

$$
\tilde{\psi}\left(x_{1}, x_{2}\right)=-\tilde{\psi}\left(-x_{1}, x_{2}\right)=\tilde{\psi}\left(x_{1},-x_{2}\right) \text { in } D\left(0, r_{0} / 2\right),
$$

we see that $\tilde{\psi}$ is given by a convergent power series expansion of the form

$$
\tilde{\psi}(x)=\sum_{j=1}^{\infty} \sum_{k=0}^{\infty} d_{j, k} x_{1}^{2 j-1} x_{2}^{2 k} \quad \text { in } D\left(0, r_{0} / 2\right) \text {. }
$$

Rewriting this in the polar coordinate, we have

$$
\tilde{\psi}(x)=\sum_{j=1}^{\infty} \sum_{k=1}^{j} C_{j, k}^{+} r^{2 j-1} \cos (2 k-1) \theta \quad \text { in } \quad D\left(0, r_{0} / 2\right) .
$$

Let us show that $C_{1,1}^{+}>0$. Note that $\tilde{\psi}=\Psi_{0}^{+}$in $D_{++}\left(r_{0} / 2\right)$. Because $\Psi_{0}^{+}>0$ in $\Omega_{+}$and $\cos \theta>0$ on $(0, \pi / 2)$, we have $C_{1,1}^{+} \geq 0$. Seeking a contradiction, we assume that $C_{1,1}^{+}=0$. Inserting (1.8) into (1.5) and using the analiticity of $\tilde{\psi}$ in $D\left(0, r_{0} / 2\right)$, we have the recurrent formula

$$
C_{j+1, k}^{+}=-\frac{\lambda_{0}}{4(j+k)(j+1-k)} C_{j, k}^{+}
$$

for $j \geq 1$ and $1 \leq k \leq j$. Let us show that for all $j \in \mathbf{N}^{*}$,

$$
C_{j, k}^{+}=0 \quad \text { for } \quad 1 \leq k \leq j
$$

by using induction on $j$. (1.10) is valid for $j=1$ by the assumption. Let $m \in \mathbf{N}^{*}$. Suppose that (1.10) is valid for $j \leq m$. Then (1.9) implies that $C_{m+1, k}^{+}=0$ for $1 \leq k \leq m$. So, (1.8) 
implies that

$$
\tilde{\psi}(x)=C_{m+1, m+1}^{+} r^{2 m+1} \cos (2 m+1) \theta+\mathcal{O}\left(r^{2 m+3}\right)
$$

as $r \rightarrow 0$. Because $\Psi_{0}^{+}>0$ in $\Omega_{+}$and $\cos (2 m+1) \theta$ takes both positive and negative sign on $(0, \pi / 2)$, we get $C_{m+1, m+1}^{+}=0$. Hence (1.10) is also valid for $j \leq m+1$. Therefore (1.10) holds for all $j \in \mathbf{N}^{*}$. This together with the analiticity of $\tilde{\psi}$ in $D\left(0, r_{0} / 2\right)$ implies that $\tilde{\psi}=0$ in $D\left(0, r_{0} / 2\right)$. But this violates the fact that $\Psi_{0}^{+}>0$ in $\Omega_{+}$. Hence we get $C_{1,1}^{+}>0$. This completes the proof of Proposition 1.1.

Next we introduce function spaces which we need on the sequel. For an open set $\Sigma$ in $\mathbf{R}^{2}$, a finite subset $S$ of $\partial \Sigma$, and $k \in \mathbf{N} \cup\{\infty\}$, we define

$$
\begin{array}{r}
C^{k}(\bar{\Sigma} \backslash S)=\left\{u: \Sigma \rightarrow \mathbf{R} \mid u \in C^{k}(\overline{\Sigma \backslash A}) \text { for any open set } A\right. \\
\text { such that } S \subset A \text { and } \Sigma \backslash A \neq \emptyset\} .
\end{array}
$$

Choose $\chi \in C^{\infty}([0, \infty))$ such that

$$
\chi(r)=1 \quad \text { on } \quad\left[0, r_{0} / 4\right], \quad \chi(r)=0 \quad \text { on } \quad\left[r_{0} / 2, \infty\right) .
$$

For $p \in \mathbf{N}$, we define $J_{p}^{ \pm}$as the class of functions $u$ which satisfies the conditions (a), (b), (c), and (d) below.
(a) $u \in C^{\infty}\left(\overline{\Omega_{ \pm}} \backslash\left\{0, \gamma\left(t_{0}\right)\right\}\right),(1-\chi(r)) u \in \operatorname{Dom}\left(L^{ \pm}\right)$.
(b) $u=0$ on $\partial \Omega \cap \partial \Omega_{ \pm}, \frac{\partial}{\partial n} u=0$ on $\gamma\left(\left(0, t_{0}\right)\right)$.
(c) The function $u$ admits an asymptotic expansion of the form

$$
\begin{aligned}
u(x) \sim & \left(\sum_{s=0}^{p-1} \sum_{j=p-s}^{p}+\sum_{s=p}^{2 p-2} \sum_{j=1}^{p}+\sum_{s=2 p-1}^{\infty} \sum_{j=p-s}^{0}\right) a_{s, j} r^{-2 p+2 s+1} \cos (2 j-1) \theta \\
& +\sum_{s=p}^{\infty} \sum_{j=1}^{\min \{p, s-p+1,[(s+1) / 2]\}} b_{s, j} r^{2 s-2 p+1} \log r \cos (2 j-1) \theta
\end{aligned}
$$

as $r \rightarrow 0$ and $x \in \Omega_{ \pm}$, which can be differentiated term by term infinitely many times.

(d) There exists $r_{1} \in\left(0, r_{0}\right)$ such that for each $N \geq 2 p$ the function

$$
\begin{aligned}
u(x)- & \left(\sum_{s=0}^{p-1} \sum_{j=p-s}^{p}+\sum_{s=p}^{2 p-2} \sum_{j=1}^{p}+\sum_{s=2 p-1}^{N} \sum_{j=p-s}^{0}\right) a_{s, j} r^{-2 p+2 s+1} \cos (2 j-1) \theta \\
& -\sum_{s=p}^{N} \sum_{j=1}^{\min \{p, s-p+1,[(s+1) / 2]\}} b_{s, j} r^{2 s-2 p+1} \log r \cos (2 j-1) \theta
\end{aligned}
$$

in $\Omega_{ \pm} \cap D\left(0, r_{1}\right)$ extends to a function $h_{N}^{ \pm}(x) \in C^{2 N-4 p+3}\left(\overline{D\left(0, r_{1}\right)}\right)$ which is even in $x_{2}$ and is odd in $x_{1}$. 
For $f, g \in \cap_{r \in\left(0, r_{0}\right)} L^{2}\left(\Omega_{ \pm} \backslash D(0, r)\right)$, we define

$$
(f, g)_{\Omega_{ \pm}}=\lim _{r \rightarrow+0}(f, g)_{L^{2}\left(\Omega_{ \pm} \backslash D(0, r)\right)}
$$

if the limit exists.

The following lemma solves the outer equations.

Lemma 1.2. Let $p \in \mathbf{N}$ and $f \in J_{p}^{ \pm}$. Given $\left\{a_{j}\right\}_{j=0}^{p} \subset \mathbf{R}$, there exists a unique $\mu \in \mathbf{R}$ for which the equation

$$
\left\{\begin{array}{l}
\left(\Delta+\lambda_{0}\right) \varphi=-\mu \Psi_{0}^{ \pm}+f \quad \text { in } \quad \Omega_{ \pm}, \\
\varphi=0 \quad \text { on } \partial \Omega \cap \partial \Omega_{ \pm}, \quad \frac{\partial}{\partial n} \varphi=0 \quad \text { on } \quad \gamma\left(\left(0, t_{0}\right)\right), \\
\left(\varphi, \Psi_{0}^{ \pm}\right)_{\Omega_{ \pm}}=0
\end{array}\right.
$$

has a solution $\varphi(x) \in J_{p+1}^{ \pm}$whose asymptotic expansion as $r \rightarrow 0$ :

$$
\begin{aligned}
\varphi(x) \sim & \left(\sum_{s=0}^{p} \sum_{j=p+1-s}^{p+1}+\sum_{s=p+1}^{2 p} \sum_{j=1}^{p+1}+\sum_{s=2 p+1}^{\infty} \sum_{j=p+1-s}^{0}\right) E_{s, j} r^{-2 p+2 s-1} \cos (2 j-1) \theta \\
& +\sum_{s=p+1}^{\infty} \sum_{j=1}^{\min \{p+1, s-p,[(s+1) / 2]\}} F_{s, j} r^{2 s-2 p-1} \log r \cos (2 j-1) \theta
\end{aligned}
$$

satisfies

$$
E_{s, p+1-s}=a_{s} \quad \text { for } \quad 0 \leq s \leq p .
$$

Moreover, we have

$$
\mu=\left(f, \Psi_{0}^{ \pm}\right)_{\Omega_{ \pm}}+\sum_{s=0}^{p} \frac{\pi}{2}(-2 p+2 s-1) a_{s} C_{p+1-s, p+1-s}^{ \pm} .
$$

ProOF. We shall demonstrate the assertion only for $(1.11)_{+}$, because that for $(1.11)_{-}$ is similar.

Since $f \in J_{p}^{+}$, the function $f$ admits the asymptotic expansion of the form

$$
\begin{aligned}
f(x) \sim & \left(\sum_{s=0}^{p-1} \sum_{j=p-s}^{p}+\sum_{s=p}^{2 p-2} \sum_{j=1}^{p}+\sum_{s=2 p-1}^{\infty} \sum_{j=p-s}^{0}\right) a_{s, j} r^{-2 p+2 s+1} \cos (2 j-1) \theta \\
& +\sum_{s=p}^{\infty} \sum_{j=1}^{\min \{p, s-p+1,[(s+1) / 2]\}} b_{s, j} r^{2 s-2 p+1} \log r \cos (2 j-1) \theta
\end{aligned}
$$


as $r \rightarrow 0$. For $N \geq 2 p$, we introduce the partial sum of the formal power series on the right side of (1.13):

$$
\begin{aligned}
F_{N}(x)= & \left(\sum_{s=0}^{p-1} \sum_{j=p-s}^{p}+\sum_{s=p}^{2 p-2} \sum_{j=1}^{p}+\sum_{s=2 p-1}^{N} \sum_{j=p-s}^{0}\right) a_{s, j} r^{-2 p+2 s+1} \cos (2 j-1) \theta \\
& +\sum_{s=p}^{N} \sum_{j=1}^{\min \{p, s-p+1,[(s+1) / 2]\}} b_{s, j} r^{2 s-2 p+1} \log r \cos (2 j-1) \theta .
\end{aligned}
$$

Thanks to the formulae

$$
\begin{aligned}
\Delta\left(r^{2 j+2 m-1} \cos (2 j-1) \theta\right)= & 4 m(2 j+m-1) r^{2 j+2 m-3} \cos (2 j-1) \theta, \\
\Delta\left(r^{2 j+2 m-1} \log r \cos (2 j-1) \theta\right)= & 4 m(2 j+m-1) r^{2 j+2 m-3} \log r \cos (2 j-1) \theta \\
& +2(2 j+2 m-1) r^{2 j+2 m-3} \cos (2 j-1) \theta,
\end{aligned}
$$

one can construct the power series

$$
\begin{aligned}
\Psi_{N}(x)= & \left(\sum_{s=0}^{p} \sum_{j=p+1-s}^{p+1}+\sum_{s=p+1}^{2 p} \sum_{j=1}^{p+1}+\sum_{s=2 p+1}^{N+1} \sum_{j=p+1-s}^{0}\right) \hat{E}_{s, j} r^{-2 p+2 s-1} \cos (2 j-1) \theta \\
& +\sum_{s=p+1}^{N+1} \sum_{j=1}^{\min \{p+1, s-p,[(s+1) / 2]\}} \hat{F}_{s, j} r^{2 s-2 p-1} \log r \cos (2 j-1) \theta
\end{aligned}
$$

that satisfies $\left(\Delta+\lambda_{0}\right) \Psi_{N}=F_{N}$ and

$$
\hat{E}_{s, p+1-s}=a_{s} \text { for } 0 \leq s \leq p .
$$

We seek the solution of $(1.11)_{+}$in the form

$$
\varphi(x)=\chi(r) \Psi_{N}(x)+\tau_{N}(x), \quad \tau_{N} \in \operatorname{Dom}\left(L^{+}\right) .
$$

Plugging this into the equation $(1.11)_{+}$, we obtain the equation for $\tau_{N}$ :

$$
\left\{\begin{array}{l}
\left(\Delta+\lambda_{0}\right) \tau_{N}=-\mu \Psi_{0}^{+}+g_{N} \quad \text { in } \Omega_{+}, \\
\tau_{N}=0 \quad \text { on } \partial \Omega \cap \partial \Omega_{+}, \quad \frac{\partial}{\partial n} \tau_{N}=0 \quad \text { on } \quad \gamma\left(\left(0, t_{0}\right)\right), \\
\left(\tau_{N}, \Psi_{0}^{+}\right)_{L^{2}\left(\Omega_{+}\right)}=-\left(\chi(r) \Psi^{N}(x), \Psi_{0}^{+}\right)_{\Omega_{+}},
\end{array}\right.
$$

where

$$
g_{N}=(1-\chi) f-\Psi_{N} \Delta \chi-2 \nabla \chi \cdot \nabla \Psi_{N}+\chi\left(f-F_{N}\right) .
$$

Since $g_{N} \in L^{2}\left(\Omega_{+}\right)$and since $\lambda_{1}^{+}$is a simple eigenvalue of $L^{+}$, we infer that the equation (1.17) has a solution in $\operatorname{Dom}\left(L^{+}\right)$if and only if

$$
\left(-\mu \Psi_{0}^{ \pm}+g_{N}, \Psi_{0}^{+}\right)_{L^{2}\left(\Omega_{+}\right)}=0 ; \quad \text { i.e. } \quad \mu=\left(g_{N}, \Psi_{0}^{+}\right)_{L^{2}\left(\Omega_{+}\right)} .
$$


We define $\mu_{N}=\left(g_{N}, \Psi_{0}^{+}\right)_{L^{2}\left(\Omega_{+}\right)}$. For $\mu=\mu_{N}$, we denote the unique solution of (1.17) in $\operatorname{Dom}\left(L^{+}\right)$by $\tau_{N}$ again. We put

$$
\varphi_{N}(x)=\chi(r) \Psi_{N}(x)+\tau_{N}(x) .
$$

Let us show that both $\mu_{N}$ and $\varphi_{N}$ are independent of the choice of $N \geq 2 p$. For $N, M \geq$ $2 p$, we have

$$
\begin{gathered}
\left(\Delta+\lambda_{0}\right)\left(\varphi_{N}-\varphi_{M}\right)=\left(\mu_{N}-\mu_{M}\right) \Psi_{0}^{+} \quad \text { in } \quad \Omega_{+}, \\
\left(\varphi_{N}-\varphi_{M}, \Psi_{0}^{+}\right)_{\Omega_{+}}=0, \\
\varphi_{N}(x)-\varphi_{M}(x)=\chi(r)\left(\Psi_{N}(x)-\Psi_{M}(x)\right)+\tau_{N}(x)-\tau_{M}(x) \in \operatorname{Dom}\left(L^{+}\right) .
\end{gathered}
$$

Since $\lambda_{0}$ is a simple eigenvalue of $L^{+}$, we get $\mu_{N}-\mu_{M}=0$ and $\varphi_{N}-\varphi_{M}=0$. Thus both $\mu_{N}$ and $\varphi_{N}$ are independent of the choice of $N \geq 2 p$, which we denote by $\mu$ and $\varphi$, respectively.

Next we compute the asymptotic expansion of $\tau_{N}(x)$ as $r \rightarrow 0$. Since $f \in J_{p}^{+}$, there exist $r_{1} \in\left(0, r_{0}\right)$ and $h \in C^{2 N-4 p+3}\left(\overline{D\left(0, r_{1}\right)}\right)$ such that

$$
\begin{gathered}
-\mu \Psi_{0}^{+}+g_{N}=h \quad \text { on } \quad \Omega_{+} \cap D\left(0, r_{1}\right), \\
h\left(x_{1}, x_{2}\right)=h\left(x_{1},-x_{2}\right)=-h\left(-x_{1}, x_{2}\right) \quad \text { in } D\left(0, r_{1}\right) .
\end{gathered}
$$

Applying the reflection argument in the proof of Proposition 1.1 to (1.17), we see that $\left.\tau_{N}\right|_{\Omega_{+} \cap D\left(0, r_{1}\right)}$ extends to a function $\hat{\tau}_{N}(x) \in H^{2 N-4 p+5}\left(D\left(0, r_{1} / 2\right)\right)$ which is even in $x_{2}$ and is odd in $x_{1}$. By the Sobolev imbedding theorem we claim that $\hat{\tau}_{N}(x) \in$ $C^{2 N-4 p+3}\left(\overline{D\left(0, r_{1} / 4\right)}\right)$. Hence the function $\hat{\tau}_{N}$ admits the asymptotic expansion of the form

$$
\partial_{x}^{\beta}\left(\hat{\tau}_{N}(x)-\sum_{j=1}^{N-2 p+1} \sum_{k=1}^{j} S_{j, k} r^{2 j-1} \cos (2 k-1) \theta\right)=\mathcal{O}\left(r^{2 N-4 p+3-|\beta|}\right)
$$

as $r \rightarrow 0$ for $|\beta| \leq 2 N-4 p+3$. Combining this with (1.18) and the independence of $\varphi_{N}$ on $N \geq 2 p$, we get the asymptotic expansion of $\varphi(x)$ as $r \rightarrow 0$.

Finally we demonstrate (1.12). By (1.11) + we have

$$
\left(\left(\Delta+\lambda_{0}\right) \varphi, \Psi_{0}^{+}\right)_{\Omega_{+}}=-\mu+\left(f, \Psi_{0}^{+}\right)_{\Omega_{+}} .
$$

We put $\Omega_{+}^{\delta}=\Omega_{+} \backslash D(0, \delta)$. Using an integration by parts, we obtain

$$
\begin{aligned}
\left(\left(\Delta+\lambda_{0}\right) \varphi, \Psi_{0}^{+}\right)_{\Omega_{+}} & =\lim _{\delta \rightarrow 0} \int_{\Omega_{+}^{\delta}} \Psi_{0}^{+}(x)\left(\Delta+\lambda_{0}\right) \varphi(x) d x \\
& =\lim _{\delta \rightarrow 0} \int_{\partial \Omega_{+}^{\delta}}\left(\Psi_{0}^{+}(x) \frac{\partial}{\partial n} \varphi(x)-\varphi(x) \frac{\partial}{\partial n} \Psi_{0}^{+}(x)\right) d S \\
& =\lim _{\delta \rightarrow 0} \int_{0}^{\pi / 2}\left(\varphi(\delta, \theta) \frac{\partial}{\partial r} \Psi_{0}^{+}(\delta, \theta)-\Psi_{0}^{+}(\delta, \theta) \frac{\partial}{\partial r} \varphi(\delta, \theta)\right) \delta d \theta .
\end{aligned}
$$


Notice that

$$
\begin{aligned}
& \left(\frac{\partial}{\partial r}\right)^{j}(\varphi(x)-\check{\varphi}(x))=\mathcal{O}\left(r^{1-j}|\log r|\right), \\
& \left(\frac{\partial}{\partial r}\right)^{j}\left(\Psi_{0}^{+}(x)-\check{\Psi}_{0}^{+}(x)\right)=\mathcal{O}\left(r^{2 p+3-j}\right)
\end{aligned}
$$

as $r \rightarrow 0$ for $j=0,1$, where

$$
\begin{gathered}
\check{\varphi}(x)=\sum_{s=0}^{p} \sum_{j=p+1-s}^{p+1} E_{s, j} r^{-2 p+2 s-1} \cos (2 j-1) \theta, \\
\check{\Psi}_{0}^{+}(x)=\sum_{j=1}^{p+1} \sum_{k=1}^{j} C_{j, k}^{+} r^{2 j-1} \cos (2 k-1) \theta .
\end{gathered}
$$

So we get

$$
\begin{aligned}
\left(\left(\Delta+\lambda_{0}\right) \varphi, \Psi_{0}^{+}\right) \Omega_{\Omega_{+}} & =\lim _{\delta \rightarrow 0} \int_{0}^{\pi / 2}\left(\check{\varphi}(\delta, \theta) \frac{\partial}{\partial r} \check{\Psi}_{0}^{+}(\delta, \theta)-\check{\Psi}_{0}^{+}(\delta, \theta) \frac{\partial}{\partial r} \check{\varphi}(\delta, \theta)\right) \delta d \theta . \\
& =-\sum_{s=0}^{p} \frac{\pi}{2}(-2 p+2 s-1) E_{s, p+1-s} C_{p+1-s, p+1-s}^{+} .
\end{aligned}
$$

Therefore we obtain (1.12).

\section{Inner equations}

In this section we solve the inner equations $(0.12)$ and $(0.17)$ by using a conformal map and a reflection argument. To this end we give a reduction scheme to solve the Poisson equation:

$$
\left\{\begin{array}{l}
\Delta \xi u(\xi)=f(\xi) \quad \text { in } \Pi_{1}, \\
u(\xi)=0 \quad \text { on } \quad\{0\} \times \mathbf{R}, \quad \frac{\partial}{\partial \xi_{2}} u(\cdot, \pm 0)=0 \quad \text { on } \quad(1, \infty)
\end{array}\right.
$$

where $f$ is a given function in $\Pi_{1}$.

For a function $g$ in $\Pi_{1}$, we define

$$
g^{ \pm}(\xi)=\frac{1}{2}\left(g\left(\xi_{1}, \xi_{2}\right) \pm g\left(\xi_{1},-\xi_{2}\right)\right), \quad \xi \in \Pi_{1}
$$

Then the functions $g^{+}\left(\xi_{1}, \cdot\right)$ and $g^{-}\left(\xi_{1}, \cdot\right)$ are even and odd, respectively, and

$$
g(\xi)=g^{+}(\xi)+g^{-}(\xi), \quad \xi \in \Pi_{1} .
$$


This decomposition reduces the equation (2.1) to the following two equations.

$$
\begin{aligned}
& \left\{\begin{array}{l}
\Delta_{\xi} u^{+}(\xi)=f^{+}(\xi) \quad \text { in } \mathbf{R}_{+}^{2}, \\
u^{+}(\xi)=0 \quad \text { on }\{0\} \times \mathbf{R}_{+}, \quad \frac{\partial}{\partial \xi_{2}} u^{+}(\cdot,+0)=0 \quad \text { on } \quad \mathbf{R}_{+} .
\end{array}\right. \\
& \left\{\begin{array}{l}
\Delta_{\xi} u^{-}(\xi)=f^{-}(\xi) \quad \text { in } \quad \mathbf{R}_{+}^{2}, \\
u^{-}(\xi)=0 \quad \text { on } \quad \Gamma_{1}, \quad \frac{\partial}{\partial n} u^{-}(\xi)=0 \quad \text { on } \Gamma_{2} .
\end{array}\right.
\end{aligned}
$$

Here $\mathbf{R}_{+}$stands for the set of all positive real numbers, $\mathbf{R}_{+}^{2}=\left(\mathbf{R}_{+}\right)^{2}, \Gamma_{2}=(1, \infty) \times\{0\}$, and $\Gamma_{1}=\partial \mathbf{R}_{+}^{2} \backslash \Gamma_{2}$.

Next we simplify the boundary condition in the latter equation by using a conformal map. We identify $\mathbf{R}^{2}$ with $\mathbf{C}$ by the map

$$
\mathbf{R}^{2} \ni\left(\xi_{1}, \xi_{2}\right) \mapsto \xi_{1}+\sqrt{-1} \xi_{2} \in \mathbf{C} .
$$

We define

$$
\xi(\eta)=\sqrt{\eta^{2}+1}, \quad \eta \in P:=\mathbf{R}_{+}+\sqrt{-1} \mathbf{R}_{+},
$$

where the branch cut of the square root is the positive real axis. It is readily seen that the map $P \ni \eta \mapsto \xi(\eta) \in P$ is conformal and it sends $\mathbf{R}_{+}$and $\sqrt{-1} \mathbf{R}_{+}$onto $\Gamma_{2}$ and $\Gamma_{1}$, respectively. Putting $v(\eta)=u^{-}(\xi(\eta))$, we arrive at the equation

$$
\left\{\begin{array}{l}
\Delta_{\eta} v(\eta)=g(\eta) \quad \text { in } \quad \mathbf{R}_{+}^{2}, \\
v(\eta)=0 \quad \text { on } \quad\{0\} \times \mathbf{R}_{+}, \quad \frac{\partial}{\partial \eta_{2}} v(\cdot, 0)=0 \quad \text { on } \quad \mathbf{R}_{+},
\end{array}\right.
$$

where

$$
g(\eta):=\frac{|\eta|^{2}}{\left|\eta^{2}+1\right|} f^{-}(\xi(\eta))
$$

Using the above procedure, we give the solutions of the Laplace equation:

$$
\left\{\begin{array}{l}
\Delta_{\xi} u(\xi)=0 \quad \text { in } \quad \Pi_{1}, \\
u(\xi)=0 \quad \text { on } \quad\{0\} \times \mathbf{R}, \quad \frac{\partial}{\partial \xi_{2}} u(\cdot, \pm 0)=0 \quad \text { on } \quad(1, \infty) .
\end{array}\right.
$$

Let $(\rho, \theta)$ be the polar coordinates of $\xi$ centered at the origin. For $j \in \mathbf{N}^{*}$, we define

$$
\begin{gathered}
U_{j}(\xi)=\operatorname{Re}\left(\xi^{2 j-1}\right)=\rho^{2 j-1} \cos (2 j-1) \theta, \\
V_{j}(\xi)=\operatorname{Re}\left(\left(\sqrt{\xi^{2}-1}\right)^{2 j-1}\right) .
\end{gathered}
$$


These functions solve the equation (2.5). Furthermore, the functions $U_{j}\left(\xi_{1}, \cdot\right)$ and $V_{j}\left(\xi_{1}, \cdot\right)$ are even and odd, respectively. A straightforward computation gives the following asymptotic expansion of $V_{j}(\xi)$, which can be differentiated term by term infinitely many times.

$$
\begin{gathered}
V_{j}(\xi) \sim \pm \sum_{l=0}^{\infty} \tau_{j, l} \rho^{2 j-2 l-1 \cos (2 j-2 l-1) \theta} \quad \text { as } \quad \rho \rightarrow \infty, \quad \xi \in \Pi_{1}, \quad \pm \xi_{2}>0, \\
\tau_{j, l}= \begin{cases}\frac{(-1)^{l}(2 j-1) ! !}{l !(2 j-2 l-1) ! 2^{l}} & \text { for } l<j, \\
\frac{(-1)^{j}(2 j-1) ! !}{j ! 2^{j}} & \text { for } l=j, \\
\frac{(-1)^{j}(2 j-1) ! !(2 l-2 j-1) ! !}{l ! 2^{l}} & \text { for } l>j .\end{cases}
\end{gathered}
$$

It is convenient to normalize the harmonic functions $V_{j}(\xi)$. We define $\tilde{V}_{j}(\xi)$ by the recurrent formulae

$$
\begin{gathered}
\tilde{V}_{1}(\xi)=V_{1}(\xi) \\
\tilde{V}_{j}(\xi)=V_{j}(\xi)-\sum_{l=1}^{j-1} \tau_{j, l} \tilde{V}_{j-l}(\xi) \text { for } \quad j \geq 2 .
\end{gathered}
$$

They admit the asymptotic expansions of the form

$$
\tilde{V}_{j}(\xi) \sim \pm \rho^{2 j-1} \cos (2 j-1) \theta \pm \sum_{l=j}^{\infty} \tilde{\tau}_{j, l} \rho^{2 j-2 l-1} \cos (2 j-2 l-1) \theta
$$

as $\rho \rightarrow \infty, \xi \in \Pi_{1}, \pm \xi_{2}>0$. These harmonic functions play an important role in the matching procedure in the next section.

Let us return to solve the equation (2.4). We denote by $\mathcal{A}$ the class of functions $w(\eta) \in$ $C^{\infty}\left(\mathbf{R}^{2} \backslash\{-\sqrt{-1}, \sqrt{-1}\}\right) \cap L_{\text {loc }}^{\infty}\left(\mathbf{R}^{2}\right)$ which is even in $\eta_{2}$ and is odd in $\eta_{1}$. We observe that if the function $g(\eta)$ extends to a function $\tilde{g}(\eta) \in \mathcal{A}$ and if $\tilde{g}(\eta)=\mathcal{O}\left(|\eta|^{-3}\right)$ as $|\eta| \rightarrow \infty$, then the Newtonian potential of $\tilde{g}$ :

$$
\tilde{v}(\eta)=\frac{1}{2 \pi} \int_{\mathbf{R}^{2}} \log |\eta-y| \tilde{g}(y) d y
$$

solves the equation (2.4), because $\tilde{v} \in \mathcal{A} \cap C^{1}\left(\mathbf{R}^{2}\right) \cap C^{\infty}\left(\mathbf{R}^{2} \backslash\{-\sqrt{-1}, \sqrt{-1}\}\right)$ and $\Delta \tilde{v}(\eta)=$ $\tilde{g}(\eta)$ in $\mathbf{R}^{2} \backslash\{-\sqrt{-1}, \sqrt{-1}\}$ (see [5, Lemmas 4.1 and 4.2]).

The following lemma gives the asymptotic expansion of $\tilde{v}$.

Lemma 2.1. Let $\tilde{g} \in \mathcal{A}$. Assume that there exists an $N \in \mathbf{N}^{*}$ such that $\tilde{g}(\eta)=$ $\mathcal{O}\left(|\eta|^{-2 N-1}\right)$ as $|\eta| \rightarrow \infty$. Then the function $\tilde{v}$ from (2.6) admits the following asymptotic 
expansion which can be differentiated term by term infinitely many times.

$$
\tilde{v}(\eta)=\sum_{\substack{1 \leq j \leq N-2 \\ j \equiv 1}} C_{j} s^{-j} \cos j \omega+\mathcal{O}\left(s^{-N+1}\right) \text { as } \quad s \rightarrow \infty
$$

Here $(s, \omega)$ stand for the polar coordinates of $\eta$ centered at the origin.

PROOF. Since

$$
|\eta-y|^{2}=s^{2}\left(1-\frac{2 y_{1} \cos \omega}{s}-\frac{2 y_{2} \sin \omega}{s}+\frac{y_{1}^{2}+y_{2}^{2}}{s^{2}}\right),
$$

the kernel of (2.6) is expressed as follows.

$$
\log |\eta-y|=\log s+\frac{1}{2} \log \left(1-\frac{2 y_{1} \cos \omega}{s}-\frac{2 y_{2} \sin \omega}{s}+\frac{y_{1}^{2}+y_{2}^{2}}{s^{2}}\right) .
$$

Let $l=N-2$. Setting

$$
H_{l}(t)=\log (1+t)-\sum_{j=1}^{l} \frac{(-1)^{j-1}}{j} t^{j} \quad \text { on } \quad(-1, \infty)
$$

we obtain

$\log |\eta-y|=\log s+\sum_{j=1}^{2 l} s^{-j} G_{j}\left(y_{1}, y_{2}, \omega\right)+\frac{1}{2} H_{l}\left(-\frac{2 y_{1} \cos \omega}{s}-\frac{2 y_{2} \sin \omega}{s}+\frac{y_{1}^{2}+y_{2}^{2}}{s^{2}}\right)$,

where $G_{j}$ is a homogeneous polynomial of $y_{1}$ and $y_{2}$ of degree $j$ whose coefficients are polynomials of $\sin \omega$ and $\cos \omega$. Plugging this expression into (2.6), we get

$$
\begin{aligned}
2 \pi \tilde{v}(\eta)= & \sum_{j=1}^{2 l} \int_{\mathbf{R}^{2}} s^{-j} G_{j}(y, \omega) \tilde{g}(y) d y \\
& +\int_{D(\eta, s / 2)} \frac{1}{2} H_{l}\left(-\frac{2 y_{1} \cos \omega}{s}-\frac{2 y_{2} \sin \omega}{s}+\frac{y_{1}^{2}+y_{2}^{2}}{s^{2}}\right) \tilde{g}(y) d y \\
& +\int_{D(\eta, s / 2)^{c}} \frac{1}{2} H_{l}\left(-\frac{2 y_{1} \cos \omega}{s}-\frac{2 y_{2} \sin \omega}{s}+\frac{y_{1}^{2}+y_{2}^{2}}{s^{2}}\right) \tilde{g}(y) d y .
\end{aligned}
$$

Notice that there exists a constant $C>0$ for which

$$
\begin{gathered}
\left|H_{l}(t)\right| \leq C|\log (1+t)| \quad \text { on } \quad\left(-1,-\frac{3}{4}\right), \\
\left|H_{l}(t)\right| \leq C|t|^{l+1} \quad \text { on } \quad\left[-\frac{3}{4}, \infty\right) .
\end{gathered}
$$


Thus we obtain

$$
\begin{aligned}
\int_{D(\eta, s / 2)} & H_{l}\left(-\frac{2 y_{1} \cos \omega}{s}-\frac{2 y_{2} \sin \omega}{s}+\frac{y_{1}^{2}+y_{2}^{2}}{s^{2}}\right) \tilde{g}(y) \mid d y \\
& \leq C \int_{D(\eta, s / 2)}\left|\log \left(1-\frac{2 y_{1} \cos \omega}{s}-\frac{2 y_{2} \sin \omega}{s}+\frac{y_{1}^{2}+y_{2}^{2}}{s^{2}}\right)\right||y|^{-2 N-1} d y \\
& \leq C \int_{D(\eta, s / 2)}(|\log | \eta-y||+\log s)|y|^{-2 N-1} d y \\
& \leq C s^{-2 N-1}\left(\int_{|\tau| \leq s / 2}|\log | \tau|| d \tau+s^{2} \log s\right) \\
& \leq C s^{-2 N+1} \log s
\end{aligned}
$$

and

$$
\begin{aligned}
\int_{D(\eta, s / 2)^{c}} & H_{l}\left(-\frac{2 y_{1} \cos \omega}{s}-\frac{2 y_{2} \sin \omega}{s}+\frac{y_{1}^{2}+y_{2}^{2}}{s^{2}}\right) \tilde{g}(y) \mid d y \\
& \leq C \int_{D(\eta, s / 2)^{c}}\left|\left(-\frac{2 y_{1} \cos \omega}{s}-\frac{2 y_{2} \sin \omega}{s}+\frac{y_{1}^{2}+y_{2}^{2}}{s^{2}}\right)\right|^{l+1}(1+|y|)^{-2 N-1} d y \\
& \leq C s^{-l-1} \int_{\mathbf{R}^{2}}(1+|y|)^{2 l+1-2 N} d y \\
& \leq C s^{-l-1} .
\end{aligned}
$$

Hence we get

$$
\tilde{v}(\eta)=\sum_{j=1}^{l} s^{-j} W_{j}(\omega)+\mathcal{O}\left(s^{-l-1}\right) \quad \text { as } \quad s \rightarrow \infty,
$$

where $W_{j}(\omega)$ is a polynomial of $\sin \omega$ and $\cos \omega$.

Applying a similar method to the derivatives of $\tilde{v}$, we get

$$
\frac{\partial^{m+n}}{\partial s^{m} \partial \omega^{n}}\left(\tilde{v}(\eta)-\sum_{j=1}^{l} s^{-j} W_{j}(\omega)\right)=\mathcal{O}\left(s^{-l-1-m}\right) \quad \text { as } \quad s \rightarrow \infty
$$

for $m, n \in \mathbf{N}$. In particular, we obtain

$$
\begin{aligned}
\Delta_{\eta} \tilde{v}(\eta) & =\sum_{j=1}^{l} \Delta_{\eta}\left(s^{-j} W_{j}(\omega)\right)+\mathcal{O}\left(s^{-l-3}\right) \\
& =\sum_{j=1}^{l} s^{-j-2}\left(j^{2} W_{j}(\omega)+W_{j}^{\prime \prime}(\omega)\right)+\mathcal{O}\left(s^{-l-3}\right) \\
& =\tilde{g}(\eta)
\end{aligned}
$$




$$
=\mathcal{O}\left(s^{-2 N-1}\right)
$$

and hence

$$
j^{2} W_{j}(\omega)+W_{j}^{\prime \prime}(\omega)=0 \quad \text { for } \quad 1 \leq j \leq l .
$$

Since $\tilde{v}(s, \pi / 2)=\frac{\partial}{\partial \omega} \tilde{v}(s, 0)=0$, we have

$$
W_{j}(\pi / 2)=W_{j}^{\prime}(0)=0 \text { for } 1 \leq j \leq l .
$$

Thus we obtain

$$
W_{j}(\omega)= \begin{cases}k_{j} \cos j \omega & \text { for odd } j \leq l \\ 0 & \text { for even } j \leq l\end{cases}
$$

where $k_{j}$ is a constant.

Our next aim is to solve the equation (2.3) when its right side is unbounded. For $q \in \mathbf{N}$, we denote by $\mathcal{B}_{q}$ the class of functions $f$ which satisfy the conditions (e) and (f) below.

(e)

$$
\frac{|\eta|^{2}}{\left|\eta^{2}+1\right|} f(\xi(\eta)) \in \mathcal{A}
$$

(f) The function $f$ admits the asymptotic expansion of the form

$$
\begin{aligned}
f(\xi) \sim & \left(\sum_{l=0}^{[(q+1) / 2]} \sum_{j=l-q}^{0}+\sum_{l=[(q+1) / 2]+1}^{q} \sum_{j=1}^{l}+\sum_{l=q+1}^{\infty} \sum_{j=l-q}^{l}\right) a_{q, l, j} \rho^{2 q-2 l+1} \cos (2 j-1) \theta \\
& +\sum_{j=1}^{[(q+1) / 2]} \sum_{l=0}^{q-2 j+1} b_{q, j, l} \rho^{2 j+2 l-1} \log \rho \cos (2 j-1) \theta
\end{aligned}
$$

as $\rho \rightarrow \infty, \xi \in P$, which can be differentiated term by term infinitely many times.

Lemma 2.2. Let $q \in \mathbf{N}$ and let $f^{-} \in \mathcal{B}_{q}$. Then the equation (2.3) has a solution $u^{-}(\xi)$ in $\mathcal{B}_{q+1}$ whose asymptotic expansion as $\rho \rightarrow \infty$ does not contain any harmonic term of positive order in $\rho$.

ProOF. Since $f^{-} \in \mathcal{B}_{q}$, we may assume that its asymptotic expansion is given by (2.7). For $N \geq q+1$, we introduce the following partial sum of (2.7):

$$
\begin{aligned}
f_{N}(\xi)= & \left(\sum_{l=0}^{[(q+1) / 2]} \sum_{j=l-q}^{0}+\sum_{l=[(q+1) / 2]+1}^{q} \sum_{j=1}^{l}+\sum_{l=q+1}^{N} \sum_{j=l-q}^{l}\right) a_{q, l, j} \rho^{2 q-2 l+1} \cos (2 j-1) \theta \\
& +\sum_{j=1}^{[(q+1) / 2]} \sum_{l=0}^{q-2 j+1} b_{q, j, l} \rho^{2 j+2 l-1} \log \rho \cos (2 j-1) \theta .
\end{aligned}
$$


Thanks to the formulae (1.15) and (1.16), one can construct a power series

$g_{N}(\xi)$

$$
\begin{aligned}
= & \left(\sum_{l=0}^{[(q+2) / 2]} \sum_{j=l-q}^{0}+\sum_{l=[(q+2) / 2]+1}^{q+1} \sum_{\substack{1 \leq j \leq l \\
j \neq q-l+2}}+\sum_{l=q+2}^{N} \sum_{j=l-q}^{l}\right) A_{q, l, j} \rho^{2 q-2 l+3} \cos (2 j-1) \theta \\
& +\sum_{j=1}^{[(q+2) / 2]} \sum_{l=0}^{q-2 j+2} B_{q, j, l} \rho^{2 j+2 l-1} \log \rho \cos (2 j-1) \theta
\end{aligned}
$$

which satisfies the equation

$$
\Delta_{\xi} g_{N}(\xi)=f_{N}(\xi) \text {. }
$$

We choose $\chi_{1} \in C^{\infty}([0, \infty))$ such that $\chi_{1}(s)=0$ for $0 \leq s \leq 2$ and $\chi_{1}(s)=1$ for $s \geq 3$. Let $\chi_{2}(\xi)=\chi_{1}(|\eta(\xi)|)$. We look for a solution of (2.3) in the form

$$
u^{-}(\xi)=\chi_{2}(\xi) g_{N}(\xi)+h_{N}(\xi) .
$$

Inserting this into (2.3), we obtain the equation for $h_{N}$ :

$$
\left\{\begin{array}{l}
\Delta_{\xi} h_{N}(\xi)=s_{N}(\xi) \quad \text { in } \quad \mathbf{R}_{+}^{2} \\
h_{N}(\xi)=0 \quad \text { on } \quad \Gamma_{1}, \quad \frac{\partial}{\partial n} h_{N}(\xi)=0 \quad \text { on } \quad \Gamma_{2}
\end{array}\right.
$$

where

$$
s_{N}=\left(1-\chi_{2}\right) f^{-}+\left(f^{-}-f_{N}\right) \chi_{2}-2 \nabla \chi_{2} \cdot \nabla g_{N}-g_{N} \Delta \chi_{2} .
$$

Putting

$$
\tilde{h}_{N}(\eta)=h_{N}(\xi(\eta)), \quad \tilde{s}_{N}(\eta)=\frac{|\eta|^{2}}{\left|\eta^{2}+1\right|} s_{N}(\xi(\eta)),
$$

we rewrite the above equation as

$$
\left\{\begin{array}{l}
\Delta_{\eta} \tilde{h}_{N}(\eta)=\tilde{s}_{N}(\eta) \quad \text { in } \quad \mathbf{R}_{+}^{2} \\
\tilde{h}_{N}(\eta)=0 \quad \text { on } \quad\{0\} \times \mathbf{R}_{+}, \quad \frac{\partial}{\partial \eta_{2}} \tilde{h}_{N}(\cdot, 0)=0 \quad \text { on } \quad \mathbf{R}_{+} .
\end{array}\right.
$$

Since $\tilde{s}_{N} \in \mathcal{A}$ and since $\tilde{s}_{N}(\eta)=\mathcal{O}\left(|\eta|^{2 q-2 N-1}\right)$, we infer by Lemma 2.1 that this equation has a solution $\tilde{h}_{N}$ which admits an asymptotic expansion of the form

$$
\tilde{h}_{N}(\eta)=\sum_{\substack{1 \leq j \leq N-q-2 \\ j \equiv 1}} \tilde{K}_{N, j} s^{-j} \cos j \omega+\mathcal{O}\left(s^{-[(N-q) / 2]+3}\right) \quad \text { as } \quad s \rightarrow \infty .
$$


Notice that $s^{-j} \cos j \omega$ is given by a power-series expansion of the form

$$
s^{-j} \cos j \omega=\operatorname{Re}\left(\eta(\xi)^{-j}\right)=\sum_{k=0}^{\infty} t_{j, k} \rho^{-j-2 k} \cos (j+2 k) \theta, \quad \rho \geq 2 .
$$

Therefore, the function $h_{N}(\xi)$ has an asymptotic expansion of the form

$$
\left.h_{N}(\xi)=\sum_{\substack{1 \leq j \leq N-q-2 \\ j \equiv 1}} K_{N, j} \rho^{-j} \cos j \theta+\mathcal{O}\left(\rho^{-[(N-q) / 2]+3}\right) \text { as } 2\right) \quad \rho \rightarrow \infty .
$$

Next we shall demonstrate that the right side of (2.8) is independent of the choice of $N \geq q+1$. Let $N_{1}, N_{2} \geq q+1$. The function

$$
\varphi(\xi):=\left(\chi_{2}(\xi) g_{N_{1}}(\xi)+u_{N_{1}}(\xi)\right)-\left(\chi_{2}(\xi) g_{N_{2}}(\xi)+u_{N_{2}}(\xi)\right)
$$

is harmonic and bounded in $P$. So the function $\varphi(\xi(\eta))$ extends to a bounded, harmonic function in $\mathbf{R}^{2}$. Thus the Liouville theorem implies that $\varphi$ identically equals zero.

\section{Construction of the approximate second eigenvalue}

This section is devoted to the construction of the coefficients in $(0.8),(0.9)_{ \pm}$, and $(0.10)$. We first give the explicit form of their leading terms. Put

$$
\varphi_{0,0}^{ \pm}(x)=k_{ \pm} \Psi_{0}^{ \pm}(x), \quad x \in \Omega_{ \pm},
$$

$k_{ \pm}$being a constant which we shall specify later. Using $(0.2)$ and $\xi=\varepsilon^{-1} x$, we have

$$
\begin{aligned}
\varphi_{0,0}^{ \pm}(x) & =k_{ \pm} C_{1,1}^{ \pm} r \cos \theta+\mathcal{O}\left(r^{3}\right) \\
& =\varepsilon k_{ \pm} C_{1,1}^{ \pm} \rho \cos \theta+\mathcal{O}\left(r^{3}\right) \quad \text { as } \quad r \rightarrow 0 .
\end{aligned}
$$

Recall that $v_{1,0}(\xi)$ is the coefficient of $\varepsilon$ in $(0.10)$ and is harmonic in $\Pi_{1}$. So we seek $v_{1,0}(\xi)$ for which

$$
v_{1,0}(\xi)=k_{ \pm} C_{1,1}^{ \pm} \rho \cos \theta+o(\rho) \quad \text { as } \quad \rho \rightarrow \infty, \quad \pm \xi_{2}>0 .
$$

Such a harmonic function is given by

$$
v_{1,0}(\xi)=\frac{1}{2}\left(k_{+} C_{1,1}^{+}+k_{-} C_{1,1}^{-}\right) U_{1}(\xi)+\frac{1}{2}\left(k_{+} C_{1,1}^{+}-k_{-} C_{1,1}^{-}\right) V_{1}(\xi) .
$$

We have

$$
\begin{aligned}
\varepsilon v_{1,0}(\xi) & =\varepsilon k_{ \pm} C_{1,1}^{ \pm} \rho \cos \theta \mp \varepsilon \frac{1}{4}\left(k_{+} C_{1,1}^{+}-k_{-} C_{1,1}^{-}\right) \rho^{-1} \cos \theta+\varepsilon \mathcal{O}\left(\rho^{-3}\right) \\
& =\varepsilon k_{ \pm} C_{1,1}^{ \pm} \rho \cos \theta \mp \varepsilon^{2} \frac{1}{4}\left(k_{+} C_{1,1}^{+}-k_{-} C_{1,1}^{-}\right) r^{-1} \cos \theta+\varepsilon \mathcal{O}\left(\rho^{-3}\right)
\end{aligned}
$$


as $\rho \rightarrow \infty, \pm \xi_{2}>0$. Since $\varphi_{1,0}^{ \pm}$is the coefficient of $\varepsilon^{2}$ in $(0.9)_{ \pm}$, we look for $\varphi_{1,0}^{ \pm}$such that

$$
\varphi_{1,0}^{ \pm}(x)=\mp \frac{1}{4}\left(k_{+} C_{1,1}^{+}-k_{-} C_{1,1}^{-}\right) r^{-1} \cos \theta+o\left(r^{-1}\right)
$$

as $r \rightarrow 0$. By Lemma 1.2 we see that the solvability condition for the equation $(0.11)_{1,0}^{+}$with (3.3) + and that for $(0.11)_{1,0}^{-}$with (3.3) - are given by

$$
\lambda_{1,0}=\frac{\pi}{8 \kappa_{+}}\left(k_{+} C_{1,1}^{+}-k_{-} C_{1,1}^{-}\right) C_{1,1}^{+}
$$

and

$$
\lambda_{1,0}=-\frac{\pi}{8 \kappa_{-}}\left(k_{+} C_{1,1}^{+}-k_{-} C_{1,1}^{-}\right) C_{1,1}^{-},
$$

respectively. These two expressions coincide if and only if either

$$
\frac{k_{+}}{k_{-}}=-\frac{C_{1,1}^{+}}{C_{1,1}^{-}} \quad \text { or } \quad \frac{k_{+}}{k_{-}}=\frac{C_{1,1}^{-}}{C_{1,1}^{+}}
$$

holds. In the former case we have $\lambda_{1,0}=\frac{\pi}{8}\left(\left(C_{1,1}^{+}\right)^{2}+\left(C_{1,1}^{-}\right)^{2}\right)$, while in the latter case we get $\lambda_{1,0}=0$. We adopt the former case; we now fix

$$
k_{ \pm}= \pm C_{1,1}^{ \pm}
$$

and define $\varphi_{0,0}^{ \pm}$and $v_{1,0}$ by (3.1) and (3.2), respectively. The equation $(0.11)_{ \pm}$admits a solution $\varphi_{1,0}^{ \pm}$which satisfies $(3.3)_{ \pm}$.

By induction we shall construct the rest of the coefficients in $(0.8),(0.9)_{ \pm}$, and $(0.10)$. Our main aim in this section is to prove the following assertion.

LEMmA 3.1. There exist $\varphi_{0,0}^{ \pm},\left\{\varphi_{N+i+1, N}^{ \pm}\right\}_{N \geq 0, i \geq 0},\left\{v_{N+i+1, N}\right\}_{N \geq 0, i \geq 0}$, and $\left\{\lambda_{N+i, N}\right\}_{N \geq 0, i \geq 1}$ satisfying $(0.11)^{ \pm},(0.12)$, and the conditions (i)-(iv) below.

(i) The function $\varphi_{p+q, q}^{ \pm}$belongs to $J_{p}^{ \pm}$.

(ii) The function $v_{N+i+1, N}$ admits the decomposition

$$
v_{N+i+1, N}(\xi)=v_{N+i+1, N}^{+}(\xi)+v_{N+i+1, N}^{-}(\xi),
$$

where $v_{N+i+1, N}^{+}(\xi)$ is a linear combination of $\rho^{2 j-1} \cos (2 k-1) \theta(1 \leq j \leq i+1,1 \leq k \leq j)$, and the function $v_{N+i+1, N}^{-}$belongs to $\mathcal{B}_{i}$.

Because of these two conditions, the functions $\varphi_{p+q, q}^{ \pm}$and $v_{N+i+1, N}$ admit the asymptotic expansions of the form

$v_{N+i+1, N}(\xi) \sim \sum_{l=0}^{\infty} h_{i, l, N}^{ \pm}(\rho, \theta)+\sum_{l=1}^{i} m_{i, l, N}^{ \pm}(\rho, \theta) \log \rho \quad\left(\rho \rightarrow \infty, \quad \xi \in \Pi, \quad \pm \xi_{2}>0\right)$, 


$$
\varphi_{q+p, q}^{ \pm}(x) \sim \sum_{s=0}^{\infty} t_{s, p, q}^{ \pm}(r, \theta)+\sum_{s=p}^{\infty} u_{s, p, q}^{ \pm}(r, \theta) \log r \quad\left(r \rightarrow 0, x \in \Omega_{ \pm}\right), \quad(3.5)_{p, q}
$$

where $h_{s, l, q}^{ \pm}(\cdot, \theta), m_{s, l, q}^{ \pm}(\cdot, \theta), t_{s, l, q}^{ \pm}(\cdot, \theta)$ and $u_{s, l, q}^{ \pm}(\cdot, \theta)$ are homogeneous polynomials of degree $2 s-2 l+1$.

(iii) (matching conditions) It holds for $q \geq 0, l \geq 1$, and $s \geq l$ that

$$
u_{s, l, q}^{ \pm}(\cdot, \theta)=m_{s, l, q}^{ \pm}(\cdot, \theta) . \quad(M .1)_{s, l, q}
$$

For $q \geq 0, s \geq 0$, and $l \geq 0$, we have

$$
t_{s, l, q}^{ \pm}(\cdot, \theta)=h_{s, l, q}^{ \pm}(\cdot, \theta)-m_{s+1, l+1, q-1}^{ \pm}(\cdot, \theta) .
$$

We denote by $W_{i, N, l}$ the coefficient of $\rho^{2 i-2 l+1} \cos (2 i-2 l+1) \theta$ in the asymptotic expansion of $v_{N+i+1, N}^{-}(\xi)$ as $\rho \rightarrow \infty, 0<\theta<\frac{\pi}{2}$ for $N \geq 0, i \geq 0$, and $l \geq i+1$.

(iv) (compatibility condition) It holds for $N \geq 0$ and $M \geq 1$ that

$$
\begin{aligned}
\left(C_{1,1}^{+}\right)^{-1}\{ & -\sum_{s=0}^{M-1} \frac{\pi}{2}(2 M-2 s-1) W_{s, N, M} C_{M-s, M-s}^{+} \\
& \left.-\sum_{s=1}^{N+M-1} \sum_{j=N-s+1}^{N+M-s} \lambda_{s, N-j}\left(\varphi_{N+M-s, j}^{+}, \Psi_{0}^{+}\right)_{\Omega_{+}}\right\} \\
=-\left(C_{1,1}^{-}\right)^{-1}\left\{\sum_{s=0}^{M-1} \frac{\pi}{2}(2 M-2 s-1) W_{s, N, M} C_{M-s, M-s}^{-}\right. & \\
& \left.-\sum_{s=1}^{N+M-1} \sum_{j=N-s+1}^{N+M-s} \lambda_{s, N-j}\left(\varphi_{N+M-s, j}^{-}, \Psi_{0}^{-}\right)_{\Omega_{-}}\right\}
\end{aligned}
$$

REMARK. The number $\lambda_{M+N, N}$ is given by the both sides of $(C)_{M, N}$.

The above lemma immediately follows from the following lemma and induction.

LEMMA 3.2. Let $N \geq 0$ and $M \geq 0$. Suppose that there exist sequences

$$
\begin{aligned}
& \left\{\varphi_{N+i, N}^{ \pm}\right\}_{1 \leq i \leq M-1} \cup\left\{\varphi_{i, j}^{ \pm}\right\}_{0 \leq j \leq N-1, j+1 \leq i}, \\
& \left\{v_{N+i, N}\right\}_{1 \leq i \leq M} \cup\left\{v_{i, j}\right\}_{0 \leq j \leq N-1, j+1 \leq i}, \\
& \left\{\lambda_{N+i, N}\right\}_{1 \leq i \leq M-1} \cup\left\{\lambda_{i, j}\right\}_{0 \leq j \leq N-1, j+1 \leq i}
\end{aligned}
$$


which satisfy

$$
\begin{array}{lll}
(0.11)_{N+i, N}^{ \pm} & \text {for } 1 \leq i \leq M-1, & \\
(0.11)_{i, j}^{ \pm} & \text {for } 0 \leq j \leq N-1, \quad j+1 \leq i, \\
(0.12)_{N+i-1, N} & \text { for } 1 \leq i \leq M, \\
(0.12)_{i-1, j} & \text { for } 0 \leq j \leq N-1, \quad j+1 \leq i, \\
(M .1)_{s, l, N}^{ \pm} & \text {for } 0 \leq s \leq M-1, \quad 1 \leq l \leq s, \\
(M .1)_{s, l, q}^{ \pm} & \text {for } l \geq 1, \quad s \geq l, \quad 0 \leq q \leq N-1, \\
(M .2)_{s, l, N}^{ \pm} & \text {for } 0 \leq s \leq M-1, \quad 0 \leq l \leq M-1, \\
(M .2)_{s, l, q}^{ \pm} & \text {for } s \geq 0, \quad l \geq 0, \quad 0 \leq q \leq N-1,
\end{array}
$$

and $(C)_{M, N}$.

Then there exist $\varphi_{N+M, N}^{ \pm}, \quad v_{N+M+1, N}$ and $\lambda_{N+M, N}$ which satisfy $(0.11)_{N+M, N}^{ \pm}$, $(0.12)_{N+M, N}$,

$$
\begin{aligned}
& (M .1)_{s, l, N}^{ \pm} \quad \text { for } \quad 0 \leq s \leq M, \quad 1 \leq l \leq s, \\
& (M .2)_{s, l, N}^{ \pm} \quad \text { for } \quad 0 \leq s \leq M, \quad 0 \leq l \leq M \text {, }
\end{aligned}
$$

and $(C)_{M+1, N}$.

PROOF. We shall first solve the equation $(0.11)_{N+M, N}^{ \pm}$. We claim by Lemma 1.2 that there exists $\lambda_{M+N, N}^{ \pm}$for which the equation $(0.11)_{N+M, N}^{ \pm}$admits a solution $\tilde{\varphi}_{N+M, N}^{ \pm} \in J_{M}^{ \pm}$ such that $\left(\tilde{\varphi}_{N+M, N}^{ \pm}, \Psi_{0}^{ \pm}\right)_{\Omega_{ \pm}}=0$ and that the coefficient of $r^{-2 M+2 s+1} \cos (2 M-2 s-1) \theta$ of the asymptotic expansion of $\tilde{\varphi}_{N+M, N}^{ \pm}(x)$ as $r \rightarrow 0$ is $\pm W_{s, N, M}$ for $0 \leq s \leq M-1$. Furthermore, we have

$$
\begin{aligned}
\lambda_{M+N, N}^{ \pm}= \pm\left(C_{1,1}^{ \pm}\right)^{-1}\{ & \mp \sum_{s=0}^{M-1} \frac{\pi}{2}(2 M-2 s-1) W_{s, N, M} C_{M-s, M-s}^{ \pm} \\
& \left.-\sum_{s=1}^{N+M-1} \sum_{j=N-s+1}^{N+M-s} \lambda_{s, N-j}\left(\varphi_{N+M-s, j}^{ \pm}, \Psi_{0}^{ \pm}\right)_{\Omega_{ \pm}}\right\}
\end{aligned}
$$

By $(C)_{M, N}$ we have

$$
\lambda_{M+N, N}^{+}=\lambda_{M+N, N}^{-}=: \lambda_{M+N, N}
$$

We define

$$
\begin{gathered}
\varphi_{N+M, N}^{+}(x)=\tilde{\varphi}_{N+M, N}^{+}(x)+\tau \Psi_{0}^{+}(x) \text { for } x \in \Omega_{+}, \\
\varphi_{N+M, N}^{-}(x)=\tilde{\varphi}_{N+M, N}^{-}(x) \text { for } \quad x \in \Omega_{-},
\end{gathered}
$$

where $\tau$ is a constant which will be specified later. 
Next we solve the equation $(0.12)_{N+M, N}$. We use the reduction scheme in section 2 . By the formula (1.15) one can construct $v_{N+M+1, N}^{+}(\xi)$ which is a linear combination of $\rho^{2 j-1} \cos (2 k-1) \theta(2 \leq j \leq M+1,1 \leq k \leq j-1)$ and solves the equation

$$
\Delta_{\xi} v_{N+M+1, N}^{+}(\xi)=-\lambda_{0} v_{N+M, N}^{+}(\xi)-\sum_{m=1}^{N+M-1} \sum_{n=0}^{N} \lambda_{m, n} v_{N+M-m, N-n}^{+}(\xi) .
$$

On the other hand, Lemma 2.2 implies that the equation

$$
\left\{\begin{array}{l}
\Delta_{\xi} v_{N+M+1, N}^{-}(\xi)=-\lambda_{0} v_{N+M, N}^{-}(\xi)-\sum_{m=1}^{N+M-1} \sum_{n=0}^{N} \lambda_{m, n} v_{N+M-m, N-n}^{-}(\xi) \text { in } P, \\
v_{N+M+1, N}^{-}(\xi)=0 \quad \text { on } \quad \Gamma_{1}, \quad \frac{\partial}{\partial n} v_{N+M+1, N}^{-}(\xi)=0 \quad \text { on } \Gamma_{2}
\end{array}\right.
$$

has a solution $v_{N+M+1, N}^{-}(\xi) \in \mathcal{B}_{M}$ whose asymptotic expansion as $\rho \rightarrow \infty$ does not contain any harmonic term of positive order in $\rho$. Let $A_{l}^{ \pm}$and $B_{l}^{ \pm}$be the coefficients of $\rho^{2 M-2 l+1} \cos (2 M-2 l+1) \theta$ in the polynomials $t_{M, l, N}^{ \pm}(\rho, \theta)$ and $m_{M+1, l+1, N-1}^{ \pm}(\rho, \theta)$, respectively, for $0 \leq l \leq M$. We have $B_{l}^{-}=-B_{l}^{+}$. We define

$$
\begin{aligned}
v_{N+M+1, N}(\xi)= & v_{N+M+1, N}^{+}(\xi)+v_{N+M+1, N}^{-}(\xi) \\
& +\frac{1}{2} \sum_{l=0}^{M}\left\{\left(A_{l}^{+}+A_{l}^{-}\right) U_{M-l+1}(\xi)+\left(A_{l}^{+}-A_{l}^{-}+2 B_{l}^{+}\right) \tilde{V}_{M-l+1}(\xi)\right\}
\end{aligned}
$$

Next we verify the matching conditions. Let $0 \leq l \leq M$. Inserting (3.4) $)_{N+M, N}$ into $(0.12)_{N+M, N}$ and identifying the powers of $\rho$ and $\log \rho$, we have

$$
\begin{gathered}
\Delta_{\xi}\left(m_{M, l, N}^{ \pm}(\rho, \theta) \log \rho+h_{M, l, N}^{ \pm}(\rho, \theta)\right) \\
=-\lambda_{0}\left(m_{M-1, l, N}^{ \pm}(\rho, \theta) \log \rho+h_{M-1, l, N}^{ \pm}(\rho, \theta)\right) \\
\quad-\sum_{m=1}^{N+M-1} \sum_{n=0}^{N} \lambda_{m, n}\left(m_{M-m+n-1, l-m+n, N-n}^{ \pm}(\rho, \theta) \log \rho\right. \\
\left.\quad+h_{M-m+n-1, l-m+n, N-n}^{ \pm}(\rho, \theta)\right) .
\end{gathered}
$$

Plugging (3.5) $l, N$ into $(0.11)_{N+l, N}$ and equating the powers of $r$ and $\log r$, we get

$$
\begin{aligned}
\Delta_{x}\left(u_{M, l, N}^{ \pm}(r, \theta) \log r+t_{M, l, N}^{ \pm}(r, \theta)\right) & \\
= & -\lambda_{0}\left(u_{M-1, l, N}^{ \pm}(r, \theta) \log r+t_{M-1, l, N}^{ \pm}(r, \theta)\right)-\lambda_{N+l, N} t_{M-l-1,0,0}^{ \pm} \\
& \quad-\sum_{m=1}^{N+l-1} \sum_{n=0}^{N} \lambda_{m, n}\left(u_{n+M-m-1, n+l-m, N-n}^{ \pm}(r, \theta) \log r+t_{n+M-m-1, n+l-m, N-n}^{ \pm}(r, \theta)\right) .
\end{aligned}
$$


Inserting (3.4) $)_{N+M, N-1}$ into (0.12) $N+M, N-1$ and equating the terms of $\rho^{2 M-2 l-1} \log \rho$, we have

$$
\begin{aligned}
& \Delta \xi m_{M+1, l+1, N-1}^{ \pm}(\rho, \theta) \\
& \quad=-\lambda_{0} m_{M, l+1, N-1}^{ \pm}(\rho, \theta)-\sum_{m=1}^{M+N-1} \sum_{n=0}^{N-1} \lambda_{m, n} m_{M+n-1-m, n+l-m, N-n-1}^{ \pm}(\rho, \theta) .
\end{aligned}
$$

Combining these three equalities with the assumption on the matching conditions, we get

$$
\begin{aligned}
& \Delta_{x}\left(u_{M, l, N}^{ \pm}(r, \theta) \log r-m_{M, l, N}^{ \pm}(r, \theta) \log r\right. \\
& \left.\quad+t_{M, l, N}^{ \pm}(r, \theta)-h_{M, l, N}^{ \pm}(r, \theta)+m_{M+1, l+1, N-1}^{ \pm}(r, \theta)\right)=0 .
\end{aligned}
$$

Using the formula (1.16), we see that $\Delta_{x}\left(u_{M, l, N}^{ \pm}(r, \theta)-m_{M, l, N}^{ \pm}(r, \theta)\right)=0$ and that $\Delta_{x}\left(\left(u_{M, l, N}^{ \pm}(r, \theta)-m_{M, l, N}^{ \pm}(r, \theta)\right) \log r\right)$ is a multiple of $r^{2 M-2 l-1} \cos (2 M-2 l+1) \theta$. On the other hand, we infer that $\Delta_{x}\left(t_{M, l, N}^{ \pm}(r, \theta)-h_{M, l, N}^{ \pm}(r, \theta)+m_{M+1, l+1, N-1}^{ \pm}(r, \theta)\right)$ has no term of $r^{2 M-2 l-1} \cos (2 M-2 l+1) \theta$ in view of the formula (1.15). So we get

$$
u_{M, l, N}^{ \pm}(r, \theta)=m_{M, l, N}^{ \pm}(r, \theta)
$$

and

$$
\Delta_{x}\left(t_{M, l, N}^{ \pm}(r, \theta)-h_{M, l, N}^{ \pm}(r, \theta)+m_{M+1, l+1, N-1}^{ \pm}(r, \theta)\right)=0 .
$$

Combining the latter equality with (3.6), we arrive at

$$
t_{M, l, N}^{ \pm}(r, \theta)-h_{M, l, N}^{ \pm}(r, \theta)+m_{M+1, l+1, N-1}^{ \pm}(r, \theta)=0 .
$$

Therefore the matching conditions $(M .1)_{M, l, N}$ and $(M .2)_{M, l, N}$ are valid for $0 \leq l \leq M$. Similarly, we have $(M .2)_{s, M, N}$ for $0 \leq s \leq M-1$.

Finally we choose $\tau$ for which the compatibility condition $(C)_{M+1, N}$ hold. Notice that this condition is equivalent to

$$
\begin{aligned}
-\left(C_{1,1}^{+}\right)^{-1} \lambda_{1,0} \tau=\left(C_{1,1}^{+}\right)^{-1}\{ & \sum_{s=0}^{M-1} \frac{\pi}{2}(2 M-2 s+1) W_{s, N, M+1} C_{M+1-s, M+1-s}^{+} \\
& \left.+\sum_{s=2}^{N+M} \sum_{j=N-s+1}^{N+M+1-s} \lambda_{s, N-j}\left(\varphi_{N+M+1-s, j}^{+}, \Psi_{0}^{+}\right)_{\Omega_{+}}\right\} \\
-\left(C_{1,1}^{-}\right)^{-1}\{ & \sum_{s=0}^{M-1} \frac{\pi}{2}(2 M-2 s+1) W_{s, N, M+1} C_{M+1-s, M+1-s}^{-} \\
& \left.-\sum_{s=2}^{N+M} \sum_{j=N-s+1}^{N+M+1-s} \lambda_{s, N-j}\left(\varphi_{N+M+1-s, j}^{-}, \Psi_{0}^{-}\right)_{\Omega_{-}}\right\} .
\end{aligned}
$$


Since the right side of this equality is independent of $\tau$ and since $\lambda_{1,0}>0$, there exists $\tau$ for which this equality holds.

\section{Error estimate on approximate eigenfunction}

Let us construct an approximate eigenfunction defined in $\Omega_{\varepsilon}$ by joining the inner expansion $(0.10)$ to the outer ones $(0.9)_{ \pm}$. Choose $\chi_{0}(t) \in C^{\infty}([0, \infty))$ for which

$$
\chi_{0}(t)=1 \quad \text { on }[0,1], \quad \chi_{0}(t)=0 \quad \text { on } \quad[2, \infty) .
$$

Put

$$
\varphi_{i, j}(x)= \begin{cases}\varphi_{i, j}^{+}(x) & \text { for } \quad x \in \Omega_{+} \\ \varphi_{i, j}^{-}(x) & \text { for } \quad x \in \Omega_{-}\end{cases}
$$

For $L \in \mathbf{N}$, we define

$$
\begin{aligned}
\varphi^{L, \varepsilon}(x)= & \chi_{0}\left(\varepsilon^{-1 / 2} r\right) \sum_{k=1}^{L+1} \sum_{l=0}^{k-1} \varepsilon^{2 k-1}(\log \varepsilon)^{l} v_{k, l}\left(\varepsilon^{-1} x\right) \\
& +\left(1-\chi_{0}\left(\varepsilon^{-1 / 2} r\right)\right) \sum_{i=0}^{L} \sum_{j=0}^{i} \varepsilon^{2 i}(\log \varepsilon)^{j} \varphi_{i, j}(x) .
\end{aligned}
$$

Our aim in this section is to demonstrate the following assertion.

LEMMA 4.1. We have

$$
\varphi^{L, \varepsilon} \in \operatorname{Dom}\left(L_{\varepsilon}\right)
$$

for sufficiently small $\varepsilon>0$ and

$$
\left(\Delta+\lambda_{0}+\sum_{m=1}^{L} \sum_{n=0}^{m-1} \varepsilon^{2 m}(\log \varepsilon)^{n} \lambda_{m, n}\right) \varphi^{L, \varepsilon}=o\left(\varepsilon^{L}\right) \quad \text { in } L^{2}(\Omega) \text { as } \varepsilon \rightarrow 0 .
$$

Proof. We immediately obtain (4.1) from the following claim.

Claim. Let $n_{ \pm}$be the outward unit normal vector to $\partial \Omega_{ \pm}$. Suppose that $h \in Q_{\varepsilon}$, $\Delta h \in L^{2}(\Omega),\left.h\right|_{\Omega_{ \pm}} \in C^{2}\left(\overline{\Omega_{ \pm}} \backslash\left\{0, \gamma(\varepsilon), \gamma\left(t_{0}\right)\right\}\right)$, and $\left.\frac{\partial}{\partial n_{ \pm}} h\right|_{\Omega_{ \pm}}=0$ on $\gamma\left(\left(\varepsilon, t_{0}\right)\right)$. Then we have $h \in \operatorname{Dom}\left(L_{\varepsilon}\right)$.

We first prove this claim. Pick an $u \in Q_{\varepsilon}$. Using a standard mollifier argument together with the method in the proof of Proposition 7.1, we claim that there exist two sequences $\left\{u_{j}^{+}\right\}_{j=1}^{\infty} \subset C^{\infty}\left(\overline{\Omega_{+}}\right)$and $\left\{u_{j}^{-}\right\}_{j=1}^{\infty} \subset C^{\infty}\left(\overline{\Omega_{-}}\right)$which satisfy

(1) $\left.u_{j}^{ \pm} \rightarrow u\right|_{\Omega_{ \pm}}$in $H^{1}\left(\Omega_{ \pm}\right)$as $j \rightarrow \infty$;

(2) For each $j \in \mathbf{N}^{*}$, there exist an open set $V_{j}^{ \pm}$such that $\left(\partial \Omega \cap \partial \Omega_{ \pm}\right) \cup\{\gamma(\varepsilon)\} \subset V_{j}^{ \pm}$ and that $u_{j}^{ \pm}=0$ on $\overline{\Omega_{ \pm}} \cap V_{j}^{ \pm}$; 
(3) $u_{j}^{+}=u_{j}^{-}$on $\gamma((0, \varepsilon))$ for all $j \in \mathbf{N}^{*}$.

Put $h_{ \pm}=\left.h\right|_{\Omega_{ \pm}}$. We get

$$
\begin{aligned}
(\nabla h, \nabla u)_{L^{2}(\Omega)}= & \lim _{j \rightarrow \infty}\left\{\left(\nabla h_{+}, \nabla u_{j}^{+}\right)_{L^{2}\left(\Omega_{+}\right)}+\left(\nabla h_{-}, \nabla u_{j}^{-}\right)_{L^{2}\left(\Omega_{-}\right)}\right\} \\
= & \lim _{j \rightarrow \infty}\left\{\int_{\partial \Omega_{+}} u_{j}^{+}(x) \frac{\partial}{\partial n_{+}} h_{+}(x) d S+\int_{\partial \Omega_{-}} u_{j}^{-}(x) \frac{\partial}{\partial n_{-}} h_{-}(x) d S\right. \\
& \left.-\left(\Delta h_{+}, u_{j}^{+}\right)_{L^{2}\left(\Omega_{+}\right)}-\left(\Delta h_{-}, u_{j}^{-}\right)_{L^{2}\left(\Omega_{-}\right)}\right\} \\
= & -(\Delta h, u)_{L^{2}(\Omega)} .
\end{aligned}
$$

Hence we obtain $h \in \operatorname{Dom}\left(L_{\varepsilon}\right)$ by the definition of $\operatorname{Dom}\left(L_{\varepsilon}\right)$, and the Claim follows.

Next we prove (4.2). We have

$$
\begin{aligned}
\left(\Delta+\lambda_{0}\right. & \left.+\sum_{m=1}^{L} \sum_{n=0}^{m-1} \varepsilon^{2 m}(\log \varepsilon)^{n} \lambda_{m, n}\right) \varphi^{L, \varepsilon} \\
= & \left(1-\chi_{0}\left(\varepsilon^{-1 / 2} r\right)\right)\left\{\sum_{i=0}^{L} \sum_{j=0}^{i} \varepsilon^{2 i}(\log \varepsilon)^{j}\left(\Delta+\lambda_{0}\right) \varphi_{i, j}(x)\right. \\
& \left.+\sum_{m=1}^{L} \sum_{n=0}^{m-1} \sum_{i=0}^{L} \sum_{j=0}^{i} \varepsilon^{2 m+2 i}(\log \varepsilon)^{n+j} \lambda_{m, n} \varphi_{i, j}(x)\right\} \\
& +\chi_{0}\left(\varepsilon^{-1 / 2} r\right)\left\{\sum_{k=1}^{L+1} \sum_{l=0}^{k-1} \varepsilon^{2 k-1}(\log \varepsilon)^{l}\left(\Delta_{x}+\lambda_{0}\right) v_{k, l}\left(\varepsilon^{-1} x\right)\right. \\
& \left.+\sum_{m=1}^{L} \sum_{n=0}^{m-1} \sum_{k=1}^{L+1} \sum_{l=0}^{k-1} \varepsilon^{2 m+2 k-1}(\log \varepsilon)^{n+l} \lambda_{m, n} v_{k, l}\left(\varepsilon^{-1} x\right)\right\} \\
& +\varepsilon^{-1}\left(\Delta \chi_{0}\right)\left(\varepsilon^{-1 / 2} r\right)\left\{\sum_{k=1}^{L+1} \sum_{l=0}^{k-1} \varepsilon^{2 k-1}(\log \varepsilon)^{l} v_{k, l}\left(\varepsilon^{-1} x\right)-\sum_{i=0}^{L} \sum_{j=0}^{i} \varepsilon^{2 i}(\log \varepsilon)^{j} \varphi_{i, j}(x)\right\} \\
& +\varepsilon^{-1 / 2}\left(\nabla \chi_{0}\right)\left(\varepsilon^{-1 / 2} r\right) \cdot \nabla_{x}\left\{\sum_{k=1}^{L+1} \sum_{l=0}^{k-1} \varepsilon^{2 k-1}(\log \varepsilon)^{l} v_{k, l}\left(\varepsilon^{-1} x\right)\right. \\
& \left.+I_{L, 3}^{\varepsilon}+I_{L, 4}^{\varepsilon} \cdot \sum_{i=0}^{i} \varepsilon_{j=0}^{2 i}(\log \varepsilon)^{j} \varphi_{i, j}(x)\right\}
\end{aligned}
$$


First we estimate $I_{L, 1}^{\varepsilon}$. Using $(0.11)^{ \pm}$, we get

$$
I_{L, 1}^{\varepsilon}=\left(1-\chi_{0}\left(\varepsilon^{-1 / 2} r\right)\right) \sum_{p=L+1}^{2 L} \sum_{q=0}^{p-1} \varepsilon^{2 p}(\log \varepsilon)^{q} \sum_{m=p-L}^{L} \sum_{j=\max \{0, q-m+1\}}^{\min \{p-m, q\}} \lambda_{m, q-j} \varphi_{p-m, j} .
$$

Since $\varphi_{i, j}^{ \pm}(x)=\mathcal{O}\left(r^{-2(i-j)+1}\right)$ as $r \rightarrow 0$ and since $\varphi_{i, j}^{ \pm}(x) \in L^{2}\left(\Omega_{ \pm} \backslash D\left(0, r_{0}\right)\right)$, we get

$$
\begin{aligned}
\left\|I_{L, 1}^{\varepsilon}\right\|_{L^{2}(\Omega)} & \leq C \sum_{p=L+1}^{2 L} \sum_{q=0}^{p-1} \varepsilon^{2 p}|\log \varepsilon|^{q}\left(\int_{\varepsilon^{1 / 2} \leq r \leq r_{0}}\left(r^{-2 L+1}\right)^{2} d x\right)^{1 / 2}+C \varepsilon^{2(L+1)}|\log \varepsilon|^{L} \\
& =\mathcal{O}\left(\varepsilon^{L+3}|\log \varepsilon|^{L}\right) .
\end{aligned}
$$

Next we estimate $I_{L, 2}^{\varepsilon}$. Using (0.12), we obtain

$$
\begin{aligned}
I_{L, 2}^{\varepsilon}= & \chi_{0}\left(\varepsilon^{-1 / 2} r\right) \varepsilon^{2 L+1} \sum_{q=0}^{L}(\log \varepsilon)^{q} v_{L+1, q}\left(\varepsilon^{-1} x\right) \\
+ & \chi_{0}\left(\varepsilon^{-1 / 2} r\right) \sum_{p=L+1}^{2 L+1} \sum_{q=0}^{p-2} \varepsilon^{2 p-1}(\log \varepsilon)^{q} \\
& \times \sum_{m=\max \{p-L-1,1\}}^{\min \{p-1, L\}} \sum_{l=\max \{0, q-m+1\}}^{\min \{q, p-m-1\}} \lambda_{m, q-l} v_{p-m, l}\left(\varepsilon^{-1} x\right) .
\end{aligned}
$$

Because $\left|v_{p, q}(\xi)\right| \leq C\left(|\xi|^{2(p-q)-1}+1\right)$ on $\Pi_{1}$, we have

$$
\begin{aligned}
\left\|\chi_{0}\left(\varepsilon^{-1 / 2} r\right) v_{p, q}\left(\varepsilon^{-1} r\right)\right\|_{L^{2}(\Omega)} & \leq C\left(\int_{r \leq 2 \varepsilon^{1 / 2}}\left(\left(\varepsilon^{-1} r\right)^{4(p-q)-2}+1\right) d x\right)^{1 / 2} \\
& \leq C \varepsilon^{-(p-q)+1}
\end{aligned}
$$

and hence

$$
\left\|I_{L, 2}^{\varepsilon}\right\|_{L^{2}(\Omega)} \leq C \varepsilon^{L+1}|\log \varepsilon|^{L} .
$$

Let us estimate $I_{L, 3}^{\varepsilon}$. We rewrite $I_{L, 3}^{\varepsilon}$ as follows.

$$
\begin{aligned}
I_{L, 3}^{\varepsilon}=\varepsilon^{-1}\left(\Delta \chi_{0}\right)\left(\varepsilon^{-1 / 2} r\right)\{ & \sum_{l=0}^{L} \sum_{s=0}^{L-l} \varepsilon^{2 l+2 s+1}(\log \varepsilon)^{l} v_{l+1+s, s}(\xi) \\
& \left.-\sum_{j=0}^{L} \sum_{t=0}^{L-j} \varepsilon^{2 j+2 t}(\log \varepsilon)^{j} \varphi_{j+t, t}(x)\right\} .
\end{aligned}
$$

We put

$$
v_{l+1+s, l}^{(L-l) \pm}(\xi)=v_{l+1+s . l}(\xi)-\sum_{t=0}^{L-l} h_{s, t, l}^{ \pm}(\rho, \theta)-\sum_{t=1}^{s} m_{s, t, l}^{ \pm}(\rho, \theta) \log \rho
$$




$$
\phi_{l+t, l}^{(L-l) \pm}(x)=\varphi_{l+t, l}(x)-\sum_{s=0}^{L-l} t_{s, t, l}^{ \pm}(r, \theta)-\sum_{s=t}^{L-l} u_{s, t, l}^{ \pm}(r, \theta) \log r .
$$

Then, $(M .1)_{s, l, q}^{ \pm}$and $(M .2)_{s, l, q}^{ \pm}$imply that

$$
\begin{aligned}
I_{L, 3}^{\varepsilon}= & \varepsilon^{-1}\left(\Delta \chi_{0}\right)\left(\varepsilon^{-1 / 2} r\right)\left\{\sum_{l=1}^{L+1} \sum_{t=0}^{L-l} \varepsilon^{2 l+2 t}(\log \varepsilon)^{l} m_{L-l+1, t+1, l-1}^{ \pm}(x)\right. \\
& \left.+\sum_{l=0}^{L} \sum_{s=0}^{L-l} \varepsilon^{2 l+2 s+1}(\log \varepsilon)^{l} v_{l+1+s, s}^{(L-l) \pm}\left(\varepsilon^{-1} x\right)+\sum_{l=0}^{L} \sum_{t=0}^{L-l} \varepsilon^{2 l+2 t}(\log \varepsilon)^{l} \phi_{l+t, l}^{(L-l) \pm}(x)\right\}
\end{aligned}
$$

on $\Omega_{ \pm}$. By using

$$
\begin{gathered}
\left|\phi_{l+t, l}^{(L-l) \pm}(x)\right| \leq C r^{2 L-2 l-2 t+3}|\log r| \quad \text { in an } \quad \Omega_{ \pm} \text {-neighborhood of }\{0\} \\
\left|v_{l+1+s, l}^{(L-l) \pm}(\xi)\right| \leq C|\xi|^{2 s-2 L+2 l-1} \text { for } \xi \in \Pi, \quad \pm \xi_{2}>0, \quad|\xi| \geq 1 \\
\left|m_{L-l+1, t+1, l-1}^{ \pm}(\xi)\right| \leq C|\xi|^{2 L-2 l-2 t+1} \quad \text { for } \quad \xi \in \Pi, \quad \pm \xi_{2}>0
\end{gathered}
$$

we obtain

$$
\left\|I_{L, 3}^{\varepsilon}\right\|_{L^{2}(\Omega)}=\mathcal{O}\left(\varepsilon^{L+1 / 2}\right) .
$$

In a similar fashion, we get

$$
\left\|I_{L, 4}^{\varepsilon}\right\|_{L^{2}(\Omega)}=\mathcal{O}\left(\varepsilon^{L+1 / 2}\right) .
$$

This completes the proof of Lemma 4.1.

\section{Construction of approximate first eigenvalue}

In this section we construct the approximate first eigenvalue (0.13) and the associated approximate eigenfunctions $(0.14)_{ \pm}$and $(0.15)$, and we complete the proof of Theorem 0.1. In the case when $K \neq \emptyset$, we define

$$
\begin{gathered}
D_{0}=\left\{(s, 0,0) \in \mathbf{Z}^{3} \mid 0 \leq s\right\} \cup\left\{(s, t, 0) \in \mathbf{Z}^{3} \mid v-1 \leq s, v-1 \leq t\right\}, \\
D_{j}=\bigcup_{k=-1}^{1}\left\{(s, 3 j+v-1+k, j) \in \mathbf{Z}^{3} \mid s \geq 3 j+v-1+k\right\} \\
\cup\left\{(s, l, j) \in \mathbf{Z}^{3} \mid l \geq 3 j+v+1, s \geq 3 j+v-1\right\} \text { for } j \geq 1, \\
D=\bigcup_{j=0}^{\infty} D_{j}, \\
E_{j}=\left\{(s, t, j) \in \mathbf{Z}^{3} \mid 3 j+v+1 \leq t, s \geq t\right\} \text { for } j \geq 0,
\end{gathered}
$$




$$
E=\bigcup_{j=0}^{\infty} E_{j} .
$$

Lemma 5.1. Suppose $K \neq \varnothing$. Then there exist $\left\{\mu_{i, j}\right\}_{0 \leq j, i \geq 3 j+2 v-1},\left\{\psi_{k, 0}^{ \pm}\right\}_{k=0}^{\infty}$, $\left\{\psi_{k, l}^{ \pm}\right\}_{1 \leq l, k \geq 3 l+v-2},\left\{w_{i, 0}\right\}_{i=1}^{\infty}$, and $\left\{w_{s, q}\right\}_{1 \leq q, s \geq 3 q+v-1}$ which satisfy $\mu_{2 v-1,0} \neq 0,(0.16)_{ \pm}$, (0.17), and the following (i)-(vi).

(i) We have $\psi_{0,0}^{ \pm}=C_{1,1}^{\mp} \Psi_{0}^{ \pm}$. The functions $\psi_{v-1,0}^{ \pm}, \psi_{v, 0}^{ \pm}$, and $\psi_{3 l+v-1+q, l}^{ \pm}(l \geq 1$, $-1 \leq q \leq 1)$ are multiples of $\Psi_{0}^{ \pm}$. The functions $\psi_{3 l+v-1+q, l}^{ \pm}$belongs to $J_{q}^{ \pm}$for $l \geq 0, q \geq 2$. It holds for $1 \leq j \leq v-2$ that $\psi_{i, 0}^{ \pm}=0$.

(ii) The functions $w_{s, q}(\xi)$ admits the decomposition

$$
w_{s, q}(\xi)=w_{s, q}^{+}(\xi)+w_{s, q}^{-}(\xi), \quad \xi \in \Pi_{1} .
$$

(iii) We have

$$
\begin{aligned}
w_{j, 0}^{+}(\xi)= & \sum_{k=1}^{j} c_{j, j, k, 0} \rho^{2 j-1} \cos (2 k-1) \theta \quad \text { for } \quad 1 \leq j \leq v-1, \\
w_{\nu+s, 0}^{+}(\xi)= & \sum_{k=1}^{v+s} c_{\nu+s, v+s, k, 0} \rho^{2 s+2 v-1} \cos (2 k-1) \theta \\
& +\sum_{j=1}^{s+1} \sum_{k=1}^{j} c_{\nu+s, j, k, 0} \rho^{2 j-1} \cos (2 k-1) \theta \quad \text { for } \quad s \geq 0, \\
w_{3 q+v-1, q}^{+}=0 \quad \text { for } \quad q \geq 1, & \\
w_{3 q+v+s, q}^{+}(\xi)= & \sum_{j=1}^{s+2} \sum_{k=1}^{j} c_{3 q+v+s, j, k, q} \rho^{2 j-1} \cos (2 k-1) \theta \quad \text { for } \quad s \geq 0 \quad \text { and } \quad q \geq 1 .
\end{aligned}
$$

(iv) We have $\frac{|\eta|^{2}}{\left|\eta^{2}+1\right|} w_{s, q}^{-}(\xi(\eta)) \in \mathcal{A}$. The functions $w_{s, q}^{-}(\xi)$ admit the following asymptotic expansions as $\rho \rightarrow \infty, 0<\theta<\frac{\pi}{2}$, which can be differentiated term by term infinitely many times.

$$
\begin{gathered}
w_{j, 0}^{-}(\xi) \sim 0 \quad \text { for } \quad 1 \leq j \leq v-1 \\
w_{\nu, 0}^{-}(\xi) \sim c_{\nu,-1, v, 0}^{-} \rho^{2 v-1} \cos (2 v-1) \theta+\sum_{q=1}^{\infty} c_{\nu, q, q, 0}^{-} \rho^{1-2 q} \cos (2 q-1) \theta \\
w_{\nu+1,0}^{-}(\xi) \sim \sum_{k=1}^{v+1} c_{\nu+1,-1, k, 0}^{-} \rho^{2 v+1} \cos (2 k-1) \theta
\end{gathered}
$$




$$
\begin{aligned}
& +\sum_{k=1}^{2} c_{v+1,0, k, 0}^{-} \rho^{3} \cos (2 k-1) \theta+c_{v+1,1,1,0}^{-} \rho \cos \theta \\
& +\sum_{q=2}^{\infty} \rho^{3-2 q} \sum_{j=q-1}^{q} c_{\nu+1, q, j, 0}^{-} \cos (2 j-1) \theta \\
& w_{v+s, 0}^{-}(\xi) \sim \sum_{k=1}^{v+s} c_{\nu+s,-1, k, 0}^{-} \rho^{2 s+2 \nu-1} \cos (2 k-1) \theta \\
& +\left(\sum_{q=0}^{[(s+1) / 2]} \sum_{j=q-s}^{s}+\sum_{q=[(s+1) / 2]+1}^{s} \sum_{j=1}^{q}+\sum_{q=s+1}^{\infty} \sum_{j=q-s}^{q}\right) c_{v+s, q, j, 0}^{-} \\
& \times \rho^{2 s-2 q+1} \cos (2 j-1) \theta+\sum_{l=0}^{s-2} D_{v+s, 1, l, 0} \rho^{2 l+1} \log \rho \cos \theta \\
& +\sum_{j=2}^{[(s+1) / 2]} \sum_{l=0}^{s-2 j+1} D_{v+s, j, l, 0} \rho^{2 l+2 j-1} \log \rho \cos (2 j-1) \theta \quad \text { for } \quad s \geq 2 . \\
& w_{3 q+v-1, q}^{-}(\xi) \sim 0 \quad \text { for } \quad q \geq 1 \\
& w_{3 q+v, q}^{-}(\xi) \sim c_{3 q+v,-1,2, q}^{-} \rho^{3} \cos 3 \theta+\sum_{\substack{l=0 \\
l \neq 1}}^{\infty} c_{3 q+v, l, l, q}^{-} \rho^{1-2 l} \cos (2 l-1) \theta \quad \text { for } \quad q \geq 1 . \\
& w_{3 q+v+s, q}^{-}(\xi) \sim \sum_{k=1}^{s+2} c_{3 q+v+s,-1, k, q}^{-} \rho^{2 s+3} \cos (2 k-1) \theta \\
& +\left(\sum_{l=0}^{[(s+1) / 2]} \sum_{j=l-s}^{0}+\sum_{l=[(s+1) / 2]+1}^{s} \sum_{j=1}^{l}+\sum_{l=s+1}^{\infty} \sum_{j=l-s}^{l}\right) c_{3 q+v+s, l, j, q}^{-} \\
& \times \rho^{2 s-2 l+1} \cos (2 j-1) \theta \\
& +\sum_{l=0}^{s-2} D_{3 q+v+s, 1, l, q} \rho^{2 l+1} \log \rho \cos \theta \\
& +\sum_{j=2}^{[(s+1) / 2]} \sum_{l=0}^{s-2 j+1} D_{3 q+v+s, j, l, q} \rho^{2 l+2 j-1} \log \rho \cos (2 j-1) \theta \\
& \text { for } q \geq 1 \text { and } s \geq 1 \text {. }
\end{aligned}
$$


The above conditions (i), (ii), and (iii) allow us to write the asymptotic expansions of $\psi_{i, j}^{ \pm}(x)$ and $w_{k+1, l}(\xi)$ as follows.

$$
\begin{gathered}
\psi_{i, j}^{ \pm}(x) \sim \sum_{(s, i, j) \in D} T_{s, i, j}^{ \pm}(r, \theta)+\sum_{(s, i, j) \in E} U_{s, i, j}^{ \pm}(r, \theta) \log r \quad(r \rightarrow 0), \\
w_{k+1, l}(\xi) \sim \sum_{(k, m, l) \in D} H_{k, m, l}^{ \pm}(\rho, \theta)+\sum_{(k, m, l) \in E} M_{k, m, l}^{ \pm}(\rho, \theta) \log \rho \quad\left(\rho \rightarrow \infty, \pm \xi_{2}>0\right),
\end{gathered}
$$

where $T_{a, b, c}^{ \pm}(\cdot, \theta), U_{a, b, c}^{ \pm}(\cdot, \theta), H_{a, b, c}^{ \pm}(\cdot, \theta)$, and $M_{a, b, c}^{ \pm}(\cdot, \theta)$ are homogeneous polynomials of degree $2 a-2 b+1$.

(v) The following matching conditions are satisfied. For $(s, l, L) \in E$, we have

$$
M_{s, l, L}^{ \pm}(\cdot, \theta)=U_{s, l, L}^{ \pm}(\cdot, \theta) .
$$

For $(s, l, L) \in D$, we have

$$
H_{s, l, L}^{ \pm}(\cdot, \theta)=T_{s, l, L}^{ \pm}(\cdot, \theta)+M_{s, l, L-1}^{ \pm}(\cdot, \theta) .
$$

(vi) For $L \geq 0$ and $I \geq 0$, the following compatibility condition holds.

$$
\begin{aligned}
-\left(C_{1,1}^{-}\right)^{-1}\{ & \sum_{s=0}^{I} \frac{\pi}{2}(2 I-2 s+1) c_{3 L+v+s, I+1, I+1-s, L}^{-} C_{I+1-s, I+1-s}^{+} \\
& +\sum_{i=3 L+2 v-1}^{3 L+v+I-1} \mu_{i, L}\left(\psi_{3 L+v+I-i, 0}^{+}, \Psi_{0}^{+}\right)_{\Omega_{+}} \\
& \left.+\sum_{j=0}^{L-1} \sum_{i=3 j+2 v-1}^{I+3 j+1} \mu_{i, j}\left(\psi_{3 L+v+I-i, L-j}^{+}, \Psi_{0}^{+}\right)_{\Omega_{+}}\right\} \\
=-\left(C_{1,1}^{+}\right)^{-1}\{ & -\sum_{s=0}^{I} \frac{\pi}{2}(2 I-2 s+1) c_{3 L+v+s, I+1, I+1-s, L}^{-} C_{I+1-s, I+1-s}^{-} \\
& +\sum_{i=3 L+2 v-1}^{3 L+v+I-1} \mu_{i, L}\left(\psi_{3 L+v+I-i, 0}^{-}, \Psi_{0}^{-}\right)_{\Omega_{-}} \\
& \left.+\sum_{j=0}^{L-1} \sum_{i=3 j+2 v-1}^{I+3 j+1} \mu_{i, j}\left(\psi_{3 L+v+I-i, L-j}^{-}, \Psi_{0}^{-}\right)_{\Omega_{-}}\right\}
\end{aligned}
$$

The number $\mu_{3 L+v+I, L}$ is given by the both sides of $(c)_{I, L}$.

ProOF. We show $\mu_{2 v-1,0} \neq 0$ only, because the other statements can be shown along the lines of the proof of Lemma 3.1. We need the following explicit form of $\left\{\psi_{i, 0}^{ \pm}\right\}_{i=0}^{\nu-1}$ and $\left\{w_{j, 0}\right\}_{j=1}^{v}$ :

$$
\psi_{0,0}^{ \pm}(x)=C_{1,1}^{\mp} \Psi_{0}^{ \pm}(x)
$$




$$
\begin{aligned}
& w_{j, 0}(\xi)=C_{1,1}^{-} \sum_{k=1}^{j} C_{j, k}^{+} r^{2 j-1} \cos (2 k-1) \theta \quad \text { for } \quad 1 \leq j \leq v-1, \\
& \psi_{i, 0}^{ \pm}(x)=0 \text { for } 1 \leq i \leq v-2, \\
& \psi_{\nu-1,0}^{+}(x)=\beta \Psi_{0}^{+}(x), \\
& \psi_{\nu-1,0}^{-}(x)=0, \\
& w_{\nu, 0}(\xi)=\sum_{k=1}^{\nu-1} C_{1,1}^{-} C_{\nu, k}^{+} \rho^{2 \nu-1} \cos (2 k-1) \theta \\
& +\frac{1}{2}\left(C_{1,1}^{-} C_{v, v}^{+}-C_{1,1}^{+} C_{v, v}^{-}\right) \tilde{V}_{v}(\xi) \\
& +\frac{1}{2}\left(C_{1,1}^{-} C_{v, v}^{+}+C_{1,1}^{+} C_{v, v}^{-}\right) U_{v}(\xi) \\
& +\frac{1}{2} \beta C_{1,1}^{+}\left(U_{1}(\xi)+V_{1}(\xi)\right) \text {, }
\end{aligned}
$$

where

$$
\beta=2\left(C_{1,1}^{+}\right)^{-1}\left(C_{1,1}^{-} C_{\nu, \nu}^{+}-C_{1,1}^{+} C_{\nu, v}^{-}\right) \tilde{\tau}_{v, v} .
$$

We proceed by contradiction. Suppose that $\mu_{2 v-1,0}=0$. By the compatibility condition $(c)_{v-1,0}$ we get

$$
\begin{aligned}
0 & =-\left(C_{1,1}^{-}\right)^{-1} \sum_{s=0}^{\nu-1} \frac{\pi}{2}(2 v-2 s-1) c_{\nu+s, v, v-s, 0}^{-} C_{\nu-s, v-s}^{+} \\
& =\left(C_{1,1}^{+}\right)^{-1} \sum_{s=0}^{\nu-1} \frac{\pi}{2}(2 v-2 s-1) c_{\nu+s, v, v-s, 0}^{-} C_{\nu-s, v-s}^{-}
\end{aligned}
$$

Let us show that $c_{v, v, v, 0}^{-} \neq 0$. We identify the coefficients of $\rho^{1-2 v} \cos (2 v-1) \theta$ and $\rho^{-1} \cos \theta$ in the asymptotic expansions of the both sides of (5.1) to get

$$
\left(\begin{array}{cc}
\tilde{\tau}_{v, v} & \tau_{1,1} \\
\tilde{\tau}_{v, 2 v-1} & \tau_{1, v}
\end{array}\right)\left(\begin{array}{c}
\frac{1}{2}\left(C_{1,1}^{-} C_{\nu, v}^{+}-C_{1,1}^{+} C_{v, v}^{-}\right) \\
\frac{1}{2} \beta C_{1,1}^{+}
\end{array}\right)=\left(\begin{array}{c}
0 \\
c_{v, v, v, 0}^{-}
\end{array}\right) .
$$

The definition of $v$ implies $\frac{1}{2}\left(C_{1,1}^{-} C_{v, v}^{+}-C_{1,1}^{+} C_{v, v}^{-}\right) \neq 0$. By [3, Lemma 1] we have

$$
\operatorname{det}\left(\begin{array}{cc}
\tilde{\tau}_{v, v} & \tau_{1,1} \\
\tilde{\tau}_{v, 2 v-1} & \tau_{1, v}
\end{array}\right) \neq 0 .
$$


Therefore we have $c_{v, v, v, 0}^{-} \neq 0$. This together with (5.2) yields

$$
C_{v, v}^{ \pm} C_{1,1}^{\mp}=-\frac{2}{\pi(2 v-1) c_{v, v, v, 0}^{-}} \sum_{s=1}^{v-1} \frac{\pi}{2}(2 v-2 s-1) c_{v+s, v, v-s, 0}^{-} C_{v-s, v-s}^{ \pm} C_{1,1}^{\mp}
$$

and thus $C_{v, v}^{+} C_{1,1}^{-}=C_{v, v}^{-} C_{1,1}^{+}$which violates the definition of $\nu$. Thus we obtain $\mu_{2 v-1,0} \neq$ 0 .

We put

$$
\psi_{i, j}(x)=\left\{\begin{array}{lll}
\psi_{i, j}^{+}(x) & \text { for } & x \in \Omega_{+} \\
\psi_{i, j}^{-}(x) & \text { for } & x \in \Omega_{-}
\end{array}\right.
$$

For an integer $L \geq 2 v-1$, we define

$$
\begin{aligned}
\psi^{L, \varepsilon}= & \chi\left(\varepsilon^{-1 / 2} r\right) \sum_{k=1}^{L+1} \sum_{l=0}^{\max \{0,[(k-\nu+1) / 3]\}} \varepsilon^{2 k-1}(\log \varepsilon)^{l} w_{k, l}\left(\varepsilon^{-1} x\right) \\
& +\left(1-\chi\left(\varepsilon^{-1 / 2} r\right)\right) \sum_{i=0}^{L} \sum_{j=0}^{\max \{0,[(i-\nu+2) / 3]\}} \varepsilon^{2 i}(\log \varepsilon)^{j} \psi_{i, j}(x) .
\end{aligned}
$$

We get the following assertion.

LEMmA 5.2. We have $\psi^{L, \varepsilon} \in \operatorname{Dom}\left(L_{\varepsilon}\right)$ for sufficiently small $\varepsilon>0$ and

$$
\left(\Delta+\lambda_{0}+\sum_{i=2 v-1}^{L} \sum_{j=0}^{[(i-2 v+1) / 3]} \varepsilon^{2 i}(\log \varepsilon)^{j} \mu_{i, j}\right) \psi^{L, \varepsilon}=o\left(\varepsilon^{L}\right) \quad \text { in } \quad L^{2}(\Omega) \quad \text { as } \quad \varepsilon \rightarrow 0 .
$$

PROOF. The proof is analogous to that of Lemma 4.1.

Now we are ready to prove Theorem 0.1 .

Proof of THEOREM 0.1. We recall that for any self-adjoint operator $A$ in a real Hilbelt space $\mathcal{H}$ and for any $\mu \in \mathbf{R}$, the inequality

$$
\|(A-\mu) \varphi\|_{\mathcal{H}} \geq \operatorname{dist}(\mu, \sigma(A))\|\varphi\|_{\mathcal{H}}, \quad \varphi \in \operatorname{Dom}(A)
$$

holds. First we consider the case that $K \neq \emptyset$. Combining the above inequality with Lemmas 4.1 and 5.2 and the facts that

$$
\begin{gathered}
\left\|\psi^{L, \varepsilon}\right\|_{L^{2}(\Omega)}=\left(\left(C_{1,1}^{+}\right)^{2}+\left(C_{1,1}^{-}\right)^{2}\right)^{1 / 2}+o(1) \quad \text { as } \quad \varepsilon \rightarrow 0, \\
\left\|\varphi^{L, \varepsilon}\right\|_{L^{2}(\Omega)}=\left(\left(C_{1,1}^{+}\right)^{2}+\left(C_{1,1}^{-}\right)^{2}\right)^{1 / 2}+o(1) \quad \text { as } \quad \varepsilon \rightarrow 0,
\end{gathered}
$$

we obtain

$$
\operatorname{dist}\left(\sigma\left(L_{\varepsilon}\right), \quad \lambda_{0}+\sum_{i=2 v-1}^{L} \sum_{j=0}^{[(i-2 v+1) / 3]} \mu_{i, j} \varepsilon^{2 i}(\log \varepsilon)^{j}\right)=o\left(\varepsilon^{L}\right),
$$




$$
\operatorname{dist}\left(\sigma\left(L_{\varepsilon}\right), \quad \lambda_{0}+\sum_{m=1}^{L} \sum_{n=0}^{m-1} \lambda_{m, n} \varepsilon^{2 m}(\log \varepsilon)^{n}\right)=o\left(\varepsilon^{L}\right)
$$

for all $L \geq 2 v-1$. Because $\lambda_{j}(\cdot)$ is monotone non-decreasing, we get

$$
\lambda_{3}(\varepsilon) \geq \lambda_{3}(0)=\min \left\{\lambda_{2}^{+}, \lambda_{2}^{-}\right\}>\lambda_{0} .
$$

Using (5.3), (5.4), (5.5) and the facts that $\lambda_{1,0}>0$ and $v \geq 2$, we get

$$
\begin{gathered}
\lambda_{1}(\varepsilon)=\lambda_{0}+\sum_{i=2 v-1}^{L} \sum_{j=0}^{[(i-2 v+1) / 3]} \mu_{i, j} \varepsilon^{2 i}(\log \varepsilon)^{j}+o\left(\varepsilon^{L}\right), \\
\lambda_{2}(\varepsilon)=\lambda_{0}+\sum_{m=1}^{L} \sum_{n=0}^{m-1} \lambda_{m, n} \varepsilon^{2 m}(\log \varepsilon)^{n}+o\left(\varepsilon^{L}\right)
\end{gathered}
$$

for all $L \geq 2 v-1$. Because $\lambda_{1}(\cdot)$ is monotone non-decreasing and $\mu_{2 v-1,0} \neq 0$, we have $\mu_{2 v-1,0}>0$. So we proved (0.5) and (0.6) in the case that $K \neq \emptyset$.

Next we consider the case that $K=\emptyset$. Recall Proposition 1.1. Notice that the Taylor series expansion of $\psi_{0,0}^{+}$at $\{0\}$ coincides with that of $\psi_{0,0}^{-}$at $\{0\}$. Since the functions $\psi_{0,0}^{+}$and $\psi_{0,0}^{-}$are analytic in a neighborhood of $\{0\}$, the function

$$
\psi_{0,0}(x)=\left\{\begin{array}{lll}
\psi_{0,0}^{+}(x) & \text { for } & x \in \Omega_{+} \\
\psi_{0,0}^{-}(x) & \text { for } & x \in \Omega_{-}
\end{array}\right.
$$

is also analytic in a neighborhood of $\{0\}$ and $\psi_{0,0} \in \operatorname{Dom}\left(L_{\varepsilon}\right)$ for sufficiently small $\varepsilon \geq 0$. Moreover we have $L_{\varepsilon} \psi_{0,0}=\lambda_{0} \psi_{0,0}$ for sufficiently small $\varepsilon \geq 0$. Combining this with the fact that $\lambda_{1}(\varepsilon) \geq \lambda_{1}(0)=\lambda_{0}$, we obtain (0.7). Using (0.7), (5.4), (5.5), and the fact that $\lambda_{1,0}>0$, we get (0.5). This completes the proof of Theorem 0.1 .

\section{Proof of Theorem 0.2}

It is easily seen that the statement (ii) implies (i). So we prove the converse only. We assume (i). We put $C_{ \pm}=\partial \Omega_{ \pm} \cap \partial \Omega$. Without any loss of generality, we may assume that the curve $C_{ \pm}$is represented as follows:

$$
\begin{gathered}
C_{ \pm}:\left[0, s_{ \pm}\right] \ni t \mapsto \tau^{ \pm}(t)=\left(\tau_{1}^{ \pm}(t), \tau_{2}^{ \pm}(t)\right) \in \mathbf{R}^{2}, \\
\tau^{ \pm}(0)=0, \\
\tau^{ \pm}\left(s_{ \pm}\right)=\gamma\left(t_{0}\right)=\left(t_{0}, 0\right), \\
\left|\frac{d}{d t} \tau^{ \pm}(t)\right|=1 \quad \text { on }\left[0, s_{ \pm}\right] .
\end{gathered}
$$


Let us first show that $C_{+}$is symmetric to $C_{-}$with respect to the $x_{1}$-axis by using analytic continuation. Let $\check{C}_{-}$and $\check{\gamma}$ denote the curves

$$
\left[0, s_{-}\right] \ni t \mapsto \check{\tau}^{-}(t):=\left(\tau_{1}^{-}(t),-\tau_{2}^{-}(t)\right) \in \mathbf{R}^{2}
$$

and

$$
\left[0, t_{0}\right] \ni t \mapsto \check{\gamma}(t):=(t,-\tau(t)) \in \mathbf{R}^{2},
$$

respectively. We have $\check{\tau}^{-}(0)=0$ and $\check{\tau}^{-}\left(s_{-}\right)=\left(t_{0}, 0\right)$. Let $\check{\Omega}_{-}$be the region enclosed by the closed curve $\check{C}_{-} \cup \check{\gamma}$. We put

$$
\begin{gathered}
\psi_{+}(x)=C_{1,1}^{-} \varphi_{1}^{+}(x) \quad \text { for } \quad x \in \Omega_{+}, \\
\check{\psi}_{-}\left(x_{1}, x_{2}\right)=C_{1,1}^{+} \varphi_{1}^{-}\left(x_{1},-x_{2}\right) \quad \text { for } \quad x \in \check{\Omega}_{-} .
\end{gathered}
$$

Using Proposition 1.1 and $K=\emptyset$, we have

$$
\psi_{+}=\check{\psi}_{-} \quad \text { on } D_{++}\left(r_{0} / 2\right) \text {. }
$$

We define

$$
\begin{gathered}
S=\min \left\{s_{+}, s_{-}\right\}, \\
q=\sup \left\{t \in[0, S] \mid \quad \tau^{+}(u)=\check{\tau}^{-}(u) \quad \text { on } \quad[0, t]\right\} .
\end{gathered}
$$

By (A.2), we have $q \in(0, S]$. Let us prove that $q=S$ by contradiction. Assume that $q<S$. We have $\tau^{+}(t)=\check{\tau}^{-}(t)$ on $[0, q]$. We note that the inner unit normal vector to $\partial \Omega_{+}$at $\tau^{+}(t)$ is $\left(\frac{d}{d t} \tau_{2}^{+}(t),-\frac{d}{d t} \tau_{1}^{+}(t)\right)$. For $\eta>0$, we define a curve $\kappa_{\eta}:\left[r_{0} / 4, q\right] \rightarrow \mathbf{R}^{2}$ by

$$
\kappa_{\eta}(t)=\tau^{+}(t)+\eta\left(\frac{d}{d t} \tau_{2}^{+}(t),-\frac{d}{d t} \tau_{1}^{+}(t)\right) .
$$

Then there exists $\eta_{0}>0$ such that

$$
\kappa_{\eta}\left(\left[r_{0} / 4, q\right]\right) \subset \Omega_{+} \cap \check{\Omega}_{-} \text {for all } \eta \in\left(0, \eta_{0}\right) .
$$

Put $d=\tau^{+}(q)\left(=\check{\tau}^{-}(q)\right)$. We choose an Euclidian coordinate system $y=\left(y_{1}, y_{2}\right)$ in which

$$
\frac{d}{d t} \tau^{+}(q)=\frac{d}{d t} \check{\tau}^{-}(q)=(1,0)_{y} \quad \text { and } \quad d=O_{y}
$$

hold, where we write the coordinates in $y$ as $(\cdot, \cdot)_{y}$ and $O_{y}$ stands for the origin of the coordinate system $y$. Then there exist $r_{1}>0$ and smooth functions $f^{+}$and $\check{f}^{-}$on $\left(-r_{1}, r_{1}\right)$ such that

$$
\begin{aligned}
& \Omega_{+} \cap D\left(O_{y}, r_{1}\right)=\left\{\left(y_{1}, y_{2}\right)_{y} \in \mathbf{R}^{2} \mid y_{1} \in\left(-r_{1}, r_{1}\right), y_{2}<f^{+}\left(y_{1}\right)\right\} \cap D\left(O_{y}, r_{1}\right), \\
& \check{\Omega}_{-} \cap D\left(O_{y}, r_{1}\right)=\left\{\left(y_{1}, y_{2}\right)_{y} \in \mathbf{R}^{2} \mid y_{1} \in\left(-r_{1}, r_{1}\right), y_{2}<\check{f}^{-}\left(y_{1}\right)\right\} \cap D\left(O_{y}, r_{1}\right) .
\end{aligned}
$$


We choose $r_{2} \in\left(0, r_{1}\right)$ such that

$$
\left(y_{1}, f^{+}\left(y_{1}\right)\right)_{y} \in D\left(O_{y}, r_{1}\right) \quad \text { and } \quad\left(y_{1}, \check{f}^{-}\left(y_{1}\right)\right)_{y} \in D\left(O_{y}, r_{1}\right) \quad \text { for } \quad y_{1} \in\left[0, r_{2}\right] .
$$

Let us prove that

$$
f^{+}\left(y_{1}\right)=\check{f}^{-}\left(y_{1}\right) \quad \text { on } \quad\left(0, r_{2}\right]
$$

by contradiction. Suppose that there exists $p_{1} \in\left(0, r_{2}\right]$ such that $f^{+}\left(p_{1}\right) \neq \check{f}^{-}\left(p_{1}\right)$. We consider the case that $f^{+}\left(p_{1}\right)<\check{f}^{-}\left(p_{1}\right)$. We put $g(t)=\min \left\{f^{+}(t), \check{f}^{-}(t)\right\}$. By (6.4) and (6.5), we claim that there exists $\varepsilon_{1}>0$ such that

$$
(t, g(t)-\varepsilon)_{y} \in \Omega_{+} \cap \check{\Omega}_{-} \quad \text { for } t \in\left[0, p_{1}\right] \quad \text { and } \quad \varepsilon \in\left(0, \varepsilon_{1}\right) .
$$

We set $\eta_{2}=\min \left\{\eta_{0}, \varepsilon_{1}, r_{0} / 4\right\}$. Let $\eta \in\left(0, \eta_{2}\right)$. Note that $\psi_{+}$is analytic in $\Omega_{+}$and that $\breve{\psi}_{-}$ is analytic in $\check{\Omega}_{-}$. By using (6.1) and the analytic continuation along the continuous curve $\kappa_{\eta}\left(\left[r_{0} / 4, q\right]\right) \cup\left\{(t, g(t)-\eta)_{y} \in \mathbf{R}^{2} ; \quad t \in\left[0, p_{1}\right]\right\}\left(\subset \Omega_{+} \cap \check{\Omega}_{-}\right)$, we have $\psi_{+}=\check{\psi}_{-}$on $\left\{(t, g(t)-\eta)_{y} \in \mathbf{R}^{2} ; \quad t \in\left[0, p_{1}\right]\right\}$. Thus we get

$$
\psi_{+}=\check{\psi}_{-} \quad \text { on } \quad Y:=\left\{(t, g(t)-\eta)_{y} \in \mathbf{R}^{2} \mid t \in\left[0, p_{1}\right], \eta \in\left(0, \eta_{2}\right)\right\} .
$$

Because $\psi_{+}$and $\check{\psi}_{-}$are continuous at $h=\left(p_{1}, g\left(p_{1}\right)\right)_{y} \in \bar{Y}$, we get

$$
\psi_{+}(h)=\check{\psi}_{-}(h) \text {. }
$$

On the other hand, (6.4), (6.5), and the fact that $f^{+}\left(p_{1}\right)<\check{f}^{-}\left(p_{1}\right)$ imply that $h \in \partial \Omega_{+} \cap \check{\Omega}_{-}$. Thus we get $\psi_{+}(h)=0$ and $\check{\psi}_{-}(h)>0$ which contradict (6.7). In a similar fashion, we get a contradiction in the case that $f^{+}\left(p_{1}\right)>\check{f}^{-}\left(p_{1}\right)$. So we obtain (6.6). This combined with (6.4) and (6.5) implies that there exists $r_{3}>0$ such that $\tau^{+}(u)=\check{\tau}^{-}(u)$ on $\left[q, q+r_{3}\right]$ which violates (6.3). Thus obtain $q=S$ and hence $\tau^{+}(u)=\check{\tau}^{-}(u)$ on [0,S]. This combined with $\tau^{+}\left(s_{+}\right)=\check{\tau}^{-}\left(s_{-}\right)=\left(t_{0}, 0\right)$ and $(6.2)$ implies $\tau^{+}(S)=\check{\tau}^{-}(S)=\left(t_{0}, 0\right)$. Because $C_{+}$and $\check{C}_{-}$ are not self-intersecting, we have $S=s_{+}=s_{-}$. Hence, $C_{+}$is symmetric to $C_{-}$with respect to the $x_{1}$-axis.

Next we prove that $\tau=0$ on $\left[0, t_{0}\right]$ by contradiction. Suppose that there exists $k_{0} \in$ $\left(0, t_{0}\right)$ such that $\tau\left(k_{0}\right) \neq 0$. We consider the case that $\tau\left(k_{0}\right)>0$. We put

$$
\begin{aligned}
& k_{1}=\inf \left\{t \in\left(0, k_{0}\right) \mid \tau(t)>0 \text { on }\left[t, k_{0}\right]\right\}\left(\in\left[r_{0}, k_{0}\right)\right), \\
& k_{2}=\sup \left\{t \in\left(k_{0}, t_{0}\right) \mid \tau(t)>0 \text { on }\left[k_{0}, t\right]\right\}\left(\in\left(k_{0}, t_{0}\right]\right) .
\end{aligned}
$$

We have $\tau\left(k_{1}\right)=\tau\left(k_{2}\right)=0$ and $\tau(t)>0$ for $t \in\left(k_{1}, k_{2}\right)$. We put

$$
\Sigma_{ \pm}=\left\{(t, \pm \tau(t)) \in \mathbf{R}^{2} \mid t \in\left(k_{1}, k_{2}\right)\right\} .
$$

We denote by $M$ the domain enclosed by the closed curve $\overline{\Sigma_{+}} \cup \overline{\Sigma_{-}}$. We have

$$
M \subset \check{\Omega}_{-}, \quad \Sigma_{+} \subset \partial \Omega_{+} \cap \check{\Omega}_{-}, \quad \text { and } \quad \Sigma_{-} \subset \partial \check{\Omega}_{-} .
$$


Using similar analytic continuation as above, we have

$$
\frac{\partial}{\partial N} \psi_{+}=\frac{\partial}{\partial N} \check{\psi}_{-} \quad \text { on } \quad \Sigma_{+} \cup\left\{\left(k_{1}, 0\right)\right\}
$$

where $N$ is the inner unit normal vector to $\partial \Omega_{+}$. Hence we get

$$
\begin{gathered}
\check{\psi}_{-} \in H^{1}(M) \cap C^{\infty}\left(\bar{M} \backslash\left\{\left(k_{2}, 0\right)\right\}\right), \\
\frac{\partial}{\partial n} \check{\psi}_{-}=0 \quad \text { on } \quad \partial M \backslash\left\{\left(k_{2}, 0\right)\right\}, \\
-\Delta \check{\psi}_{-}=\lambda_{0} \check{\psi}_{-} \quad \text { in } \quad M .
\end{gathered}
$$

We put

$$
\Xi=\left\{\varphi \in C^{\infty}\left(\mathbf{R}^{2}\right) \mid \varphi=0 \text { in a neighborhood of }\left\{\left(k_{2}, 0\right)\right\}\right\} .
$$

Using (6.8), (6.9), (6.10), and Green's formula, we have

$$
\begin{aligned}
\left(\nabla \check{\psi}_{-}, \nabla \varphi\right)_{L^{2}(M)} & =\int_{\partial M} \varphi \frac{\partial}{\partial n} \check{\psi}_{-} d S-\int_{M} \varphi \Delta \check{\psi}_{-} d x \\
& =\lambda_{0}\left(\check{\psi}_{-}, \varphi\right)_{L^{2}(M)}
\end{aligned}
$$

for all $\varphi \in \Xi$. Because $\Xi$ is dense in $H^{1}(M)$ (see Proposition 7.1), (6.11) holds for all $\varphi \in H^{1}(M)$. Thus $\check{\psi}_{-}$is an eigenfunction of the Neumann Laplacian on $M$ associated with the nonzero eigenvalue $\lambda_{0}$. Hence we get $\int_{M} \breve{\psi}_{-} d x=0$ which violates the fact that $\check{\psi}_{-}>0$ on $M$. In a similar fashion, we get a contradiction in the case that $\tau\left(k_{0}\right)<0$. Thus we obtain $\tau=0$ on $\left[0, t_{0}\right]$, and the statement (ii) holds. This completes the proof of Theorem 0.2.

\section{Appendix}

PROPOSITION 7.1. Let $\Lambda \subset \mathbf{R}^{2}$ be a bounded region having the segment property and let $p \in \bar{\Lambda}$. Then

$$
\Theta=\left\{h \in C_{0}^{\infty}\left(\mathbf{R}^{2}\right) \mid h=0 \text { in a neighborhood of }\{p\}\right\}
$$

is dense in $H^{1}(\Lambda)$.

Proof. Since $\Lambda$ has the segment property, $C_{0}^{\infty}\left(\mathbf{R}^{2}\right)$ is dense in $H^{1}(\Lambda)$. So, it suffices to show that $\Theta$ is dense in $C_{0}^{\infty}\left(\mathbf{R}^{2}\right)$ with respect to the norm $\|\cdot\|_{H^{1}\left(\mathbf{R}^{2}\right)}$. For $R>e$ and $\xi \in \mathbf{R}^{2}$, we put

$$
A_{R}(\xi)= \begin{cases}\frac{1}{|\xi|^{2}(\log |\xi|) \log (\log R)} & \text { if } e \leq|\xi| \leq R \\ 0 & \text { in the other cases }\end{cases}
$$




$$
B_{R}(x)=\left(\mathcal{F}^{-1} A_{R}\right)(x-p)=\frac{1}{2 \pi} \int_{\mathbf{R}^{2}} \exp (\sqrt{-1}(x-p) \cdot \xi) A_{R}(\xi) d \xi,
$$

where $\mathcal{F}^{-1}$ stands for the inverse Fourier transformation. We obtain $B_{R} \in C^{\infty}\left(\mathbf{R}^{2}\right)$ and

$$
B_{R}(p)=\frac{1}{\log (\log R)} \int_{e}^{R} \frac{1}{r \log r} d r=1 .
$$

By the Plancherel theorem, we get

$$
\begin{aligned}
\left\|B_{R}\right\|_{H^{1}\left(\mathbf{R}^{2}\right)}^{2} & =\int_{\mathbf{R}^{2}}\left(1+|\xi|^{2}\right)\left|A_{R}(\xi)\right|^{2} d \xi \\
& \leq \frac{2 \pi}{\{\log (\log R)\}^{2}} \int_{e}^{\infty} \frac{1+r^{2}}{r^{3}(\log r)^{2}} d r \rightarrow 0 \quad \text { as } \quad R \rightarrow \infty .
\end{aligned}
$$

Let $f \in C_{0}^{\infty}\left(\mathbf{R}^{2}\right)$. We put $f_{R}(x)=\left(1-B_{R}(x)\right) f(x)$. Then we have $f_{R} \in C_{0}^{\infty}\left(\mathbf{R}^{2}\right)$ and $f_{R}(p)=0$. Moreover, we infer by (7.1) that

$$
\left\|f_{R}-f\right\|_{H^{1}\left(\mathbf{R}^{2}\right)} \rightarrow 0 \quad \text { as } \quad R \rightarrow \infty .
$$

We choose $\chi_{3} \in C_{0}^{\infty}\left(\mathbf{R}^{2}\right)$ such that $\chi_{3}(x)=1$ for $|x| \leq 1$ and that $\chi_{3}(x)=0$ for $|x| \geq 2$. For $\delta>0$, we put $f_{R, \delta}=\left(1-\chi_{3}\left(\delta^{-1}(x-p)\right)\right) f_{R}(x)$. Then we have $f_{R, \delta} \in C_{0}^{\infty}\left(\mathbf{R}^{2}\right)$ and $f_{R, \delta}=0$ on $D(p, \delta)$. Notice that

$$
\begin{aligned}
\left\|f_{R, \delta}-f_{R}\right\|_{H^{1}\left(\mathbf{R}^{2}\right)}^{2} \leq & \left\|\chi_{3}\left(\delta^{-1}(x-p)\right) f_{R}\right\|_{L^{2}\left(\mathbf{R}^{2}\right)}^{2}+2 \delta^{-2}\left\|\left(\nabla \chi_{3}\right)\left(\delta^{-1}(x-p)\right) f_{R}(x)\right\|_{L^{2}\left(\mathbf{R}^{2}\right)}^{2} \\
& +2\left\|\chi_{3}\left(\delta^{-1}(x-p)\right)\left(\nabla f_{R}\right)(x)\right\|_{L^{2}\left(\mathbf{R}^{2}\right)}^{2} .
\end{aligned}
$$

Using supp $\chi_{3}\left(\delta^{-1}(\cdot-p)\right) \subset \overline{D(0,2 \delta)}$ and $f_{R}(x)=\mathcal{O}(|x-p|)$ as $x \rightarrow p$, we have

$$
\lim _{\delta \rightarrow 0}\left\|f_{R, \delta}-f_{R}\right\|_{H^{1}\left(\mathbf{R}^{2}\right)}=0 .
$$

This combined with (7.2) implies that $\Theta$ is dense in $C_{0}^{\infty}\left(\mathbf{R}^{2}\right)$ with respect to the norm $\|\cdot\|_{H^{1}\left(\mathbf{R}^{2}\right)}$.

ACKNOWLEDGEMENT. The author thanks the referee for his/her valuable suggestions.

\section{References}

[ 1] M. DAuge and B. HelfFER, Eigenvalues variation. II. Multidimensional Problems, J. Differential Equations 104 (1993), 263-297.

[2] A. Friedman, Partial Differential Equations, Robert E. Krieger Publ. Co., Inc. (1976).

[3] R. R. GAdyL'shin, The splitting of a multiple eigenvalue in a boundary value problem for a membrane clamped to a small section of the boundary, Siberian Math. J. (3) 34 (1993), 433-450.

[ 4 ] R. R. GADYL'shin and A. M. IL'IN, Asymptotic behaviour of the eigenvalues of the Dirichlet problem in a domain with a narrow slit, Sbornik Math. 189 (1998), 503-526.

[ 5 ] D. Gilbarg and N. S. Trudinger, Elliptic partial differential equations of second order, Springer (1977). 
[6] J. HADAmard, Mémoire sur le problème d'analyse relatif à l'équilibre des plaques élastiques encastrées, Mém. Savants Etrangers 33 (1908).

[ 7 ] A. M. IL'IN, A boundary value problem for the elliptic equation of second order in a domain with a narrow slit. 1. The two-dimensional case, Math. USSR Sb. 28 (1976), 459-480.

[ 8 ] A. M. IL'IN, Matching of asymptotic expansions of solutions of boundary value problems, Translations of Mathematical Monographs, Vol. 102, American Mathematical Society (1992).

[ 9 ] T. Kato, Perturbation Theory for Linear Operators, Springer (1980).

[10] V. G. MaZ'YA, S. A. NAZAROV and B. A. Plamenevskil, Asymptotic expansions of the eigenvalues of boundary value problems for the Laplace operator in domains with small holes, Math. USSR Izv. 24 (1985), 321-345.

Present Address:

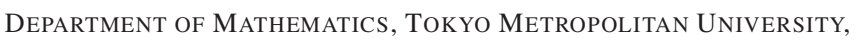
MinAMI-OHSAWA, HACHIOJI-SHI, TOKYO, 192-0397 JAPAN.

e-mail: yositomi@comp.metro-u.ac.jp 